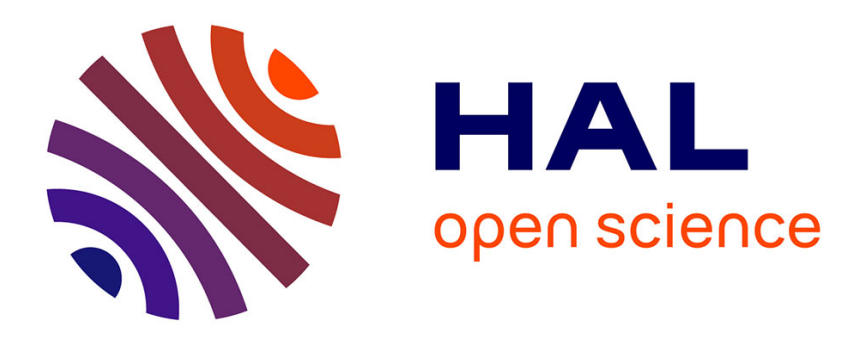

\title{
Spatial development of turbulent flow within a heated duct
}

Jérôme Hebrard, Martin Salinas-Vazquez, Olivier Métais

\section{To cite this version:}

Jérôme Hebrard, Martin Salinas-Vazquez, Olivier Métais. Spatial development of turbulent flow within a heated duct. Journal of Turbulence, 2005, 6, pp.1-20. 10.1080/14685240500103184 . hal-00265282

\section{HAL Id: hal-00265282 \\ https://hal.science/hal-00265282}

Submitted on 19 Mar 2020

HAL is a multi-disciplinary open access archive for the deposit and dissemination of scientific research documents, whether they are published or not. The documents may come from teaching and research institutions in France or abroad, or from public or private research centers.
L'archive ouverte pluridisciplinaire HAL, est destinée au dépôt et à la diffusion de documents scientifiques de niveau recherche, publiés ou non, émanant des établissements d'enseignement et de recherche français ou étrangers, des laboratoires publics ou privés. 


\title{
Spatial development of turbulent flow within a heated duct
}

\author{
J. HÉBRARD, ${ }^{\dagger}$ M. SALINAS-VASQUEZ $\ddagger$ and O. MÉTAIS $†$ \\ †Equipe MoST/LEGI/ Institut de Mécanique de Grenoble, B.P. 53, 38041 Grenoble Cedex 09, France \\ $\ddagger$ Present address: Instituto de Ingenieria, UNAM, Mexico
}

\begin{abstract}
Large eddy simulations (LES) of a turbulent field within a heated duct are presented. As opposed to a previously performed LES which assumed periodicity in the flow direction, here we consider spatially growing situations for which a thermal boundary layer grows in the longitudinal direction. Characteristic wave boundary conditions previously proposed for subsonic flows are implemented and shown to yield good results. Two different thermal boundary conditions are considered in the spatial configuration: the first case corresponds to an imposed temperature at the wall and the second to an imposed heat flux. As we move downstream in the duct, we observed the progressive formation of a unique ejection of hot fluid localized in the middle plane of the heated wall. This induces significant variations in thermal characteristics along the transverse direction of the heated wall.
\end{abstract}

\section{Introduction}

The improvement of heat transfer between a heated wall and the adjacent turbulent fluid is crucial for numerous industrial applications (heat exchangers, cooling systems in rocket engines, etc.). As far as numerical studies are concerned, statistical modelling relies heavily upon empirical laws aimed at mimicking near-wall heat exchanges. Very few Direct Numerical Simulations (DNS) or Large Eddy Simulations (LES) have been devoted to this subject. Salinas-Vázquez and Métais [1] were the first to perform a LES of turbulent flow within a straight square duct with a heated wall. The imposition of a fixed temperature at the walls was compatible with the use of periodic boundary conditions in the longitudinal direction allowing a fully developed turbulent regime to be reached. It was shown that, for a high enough temperature ratio between the heated wall and the non-heated wall, the secondary flow perpendicular to the mean flow is strongly reinforced and a unique ejection of hot fluid localized in the middle plane of the hot wall takes place.

More recently, Hébrard et al. [2] have simulated, through LES, the spatial development of turbulent flow within a straight heated duct. It is well known that the proper prescription of boundary conditions in spatially growing subsonic flows is a challenging problem which has been addressed by several authors (see e.g. [3] and [4]). After presentation of the governing equations and the numerical scheme (section 2), section 3 completes the previous work by Hébrard et al. [2] by providing a full validation of the boundary conditions implementation.

As compared with the periodic case, the spatially growing flow considered by Hébrard et al. [2] is in closer correspondence with industrial situations where a fully developed thermal regime is often not reached. As in [1], Hébrard et al. [2] considered a fixed temperature on

\footnotetext{
*Corresponding author. E-mail: Jerome.Hebrard@hmg.inpg.fr
} 
the duct walls. In several industrial devices, the heating is imposed through a constant heat flux rather than through a constant wall temperature. In section 4, we therefore present LES results where the two types of heating, constant wall temperature and constant wall heat flux, are compared.

\section{Governing equations}

We consider a Cartesian frame of reference $x, y, z$ that we will also designate as $x_{1}, x_{2}$ and $x_{3}$ for convenience. The compressible Navier-Stokes equations are written here in the so-called fast-conservation form details of which can be found in [5]:

$$
\frac{\partial U}{\partial t}+\frac{\partial F_{i}}{\partial x_{i}}=S
$$

$U$ is a five-component vector defined by:

$$
U={ }^{\mathrm{T}}\left(\rho, \rho u_{1}, \rho u_{2}, \rho u_{3}, \rho e\right)
$$

Here $\mathbf{u}=\left(u_{1}, u_{2}, u_{3}\right)$ is the velocity vector and $\rho$ is the density. In the course of this paper, we will also write $\mathbf{u}=(u, v, w)$. Equation (1) represents the evolution of density (continuity equation), momentum and total energy defined by, for an ideal gas,

$$
\rho e=\rho C_{v} T+\frac{1}{2} \rho\left(u_{1}^{2}+u_{2}^{2}+u_{3}^{2}\right)
$$

$F_{i}$ are fluxes which, $\forall i \in\{1,2,3\}$ and for a Newtonian fluid, are given by:

$$
F_{i}=\left(\begin{array}{l}
\rho u_{i} \\
\rho u_{i} u_{1}+p \delta_{i 1}-2 \mu S_{i 1} \\
\rho u_{i} u_{2}+p \delta_{i 2}-2 \mu S_{i 2} \\
\rho u_{i} u_{3}+p \delta_{i 3}-2 \mu S_{i 3} \\
(\rho e+p) u_{i}-2 \mu u_{j} S_{i j}-k \frac{\partial T}{\partial x_{i}}
\end{array}\right)
$$

$k=\rho C_{p} \kappa$ being the thermal conductivity and $\kappa$ the thermal diffusivity. $\delta_{i j}$ is the Kronecker's index and $S_{i j}$ is the deviatoric part of the deformation tensor. Neglecting bulk viscosity, $S_{i j}$ is:

$$
S_{i j}=\frac{1}{2}\left(\frac{\partial u_{i}}{\partial x_{j}}+\frac{\partial u_{j}}{\partial x_{i}}-\frac{2}{3}(\nabla \cdot \mathbf{u}) \delta_{i j}\right)
$$

Molecular viscosity is prescribed through the Sutherland empirical law (see e.g. [6]):

$$
\mu(T)=\mu\left(T_{\mathrm{ref}}\right)\left(\frac{T}{T_{\text {ref }}}\right)^{1 / 2} \frac{1+S / T_{\text {ref }}}{1+S / T}
$$

where $S, T_{\text {ref }}$ and $\mu\left(T_{\text {ref }}\right)$ are functions of the gas. The molecular conductivity $k(T)$ is obtained assuming the molecular Prandtl number is given by:

$$
\operatorname{Pr}=\frac{v}{\kappa}=\frac{C_{p} \mu(T)}{k(T)}
$$

and here is taken equal to 0.7. The classical equation of state for an ideal gas between the static pressure $p$, the temperature $T$ and the density $\rho$

$$
p=R \rho T
$$

closes the system, with $R=C_{p}-C_{v}$. We recall also that $\gamma=C_{p} / C_{v}$ is constant. 
LES techniques consist of deterministically simulating only the large scales of the flow: the LES equations are then obtained by applying a low-pass spatial filter to the Navier-Stokes equations. Scales smaller than the filter width are then filtered out, but statistically influence the large-scale motion (see [7] for details). For any quantity $f(\mathbf{x}, t)$, we designate as $\bar{f}(\mathbf{x}, t)$ the large-scale field obtained by application of a low-pass spatial filter to $f$. The derivation of the compressible LES equations is trickier than in the incompressible case. Details may be found in [8] and [9]. Indeed, application of a low-pass filter to the equations of motion, in the compressible case, leads to quantities of the following form:

$$
\overline{\rho \Phi}
$$

where $\Phi(\mathbf{x}, t)=u_{i}(\mathbf{x}, t), e(\mathbf{x}, t), T(\mathbf{x}, t)$, etc. These are not easily expressible as a simple function of $\bar{\rho}$ and $\bar{\Phi}$. To overcome this difficulty it is customary (see [10]) to introduce the density-weighted Favre average (here density-weighted filter) denoted ${ }^{\sim}$ :

$$
\tilde{\Phi}=\frac{\overline{\rho \Phi}}{\bar{\rho}}
$$

A closed system of equations for the variables $\tilde{\Phi}$ and $\bar{\rho}$ can then be derived (see [8]). In particular, the equations for $\bar{\rho} \tilde{u}_{i}$ are closed by introducing a turbulent eddy viscosity $v_{\mathrm{t}}(\boldsymbol{x}, t)$ such that:

$$
\tau_{i j}=\bar{\rho} v_{\mathrm{t}} \widetilde{S_{i j}}
$$

where $\tau_{i j}$ is the isotropic part of the subgrid stress tensor $\overline{\overline{\mathcal{T}}}$ of components

$$
\mathcal{T}_{i j}=-\overline{\rho u_{i} u_{j}}+\bar{\rho} \tilde{u}_{i} \tilde{u}_{j}
$$

The expression for $v_{t}$ used in our compressible formulation corresponds to the incompressible models described in [7]. The only difference being that $v_{t}$ is here deduced from the densityweighted filtered velocity field $\tilde{\boldsymbol{u}}$. Our subgrid-scale model is the selective structure function model (see [7] and [1] for details). The closure of the energy equation is achieved by introducing an eddy-conductivity $k_{\mathrm{t}}(\boldsymbol{x}, t)$ :

$$
k_{\mathrm{t}}(\mathbf{x}, t)=C_{p} \frac{v_{t}(\mathbf{x}, t)}{\operatorname{Pr}_{t}}
$$

where $P r_{\mathrm{t}}$ is the turbulent Prandtl number taken equal to 0.6. In the following, for the sake of simplicity, $\tilde{f}$ or $\bar{f}$ will be designated simply as $f$.

Before starting a description of the computations, one first has to define the various statistical quantities used in the article. The various statistical quantities are noted in a similar way to [1]. However, at variance with the periodic case, all statistical quantities in spatially growing simulations vary with the streamwise distance $x$. Thus, for the mean quantities, $\langle f\rangle(x, y, z)$ stands for the time average of the variable $f$; for the bulk quantities, $f_{b}(x)$ refers to the mean quantity integrated along both $y$ and $z$ directions at a given $x$-plane:

$$
f_{\mathrm{b}}(x)=\frac{1}{L y L z} \int_{0}^{L z} \int_{0}^{L y}\langle f\rangle(x, y, z) \mathrm{d} y \mathrm{~d} z
$$

$L y$ and $L z$ are the transverse dimensions of the computational domain. The bulk velocity $U_{\mathrm{b}}(x)$ involves the density and is defined as:

$$
U_{\mathrm{b}}(x)=\frac{[\rho u]_{\mathrm{b}}(x)}{\rho_{\mathrm{b}}(x)}
$$

where $\rho_{\mathrm{b}}$ is the bulk density. The fluctuation $f(\mathbf{x}, t)$ from the mean satisfies $f(\mathbf{x}, t)=$ $f(\mathbf{x}, t)-\langle f\rangle(\mathbf{x})$. We will designate $\mathbf{u}=(u, v, w)$ as the velocity vector and its components. 
For convenience, we denote $U(\mathbf{x})=\langle u\rangle(\mathbf{x}) ; V(\mathbf{x})=\langle v\rangle(\mathbf{x}) ; W(\mathbf{x})=\langle w\rangle(\mathbf{x})$. $D_{\mathrm{h}}$ stands for the duct hydraulic diameter.

\section{LES of the spatially growing duct flow: boundary conditions and computational details}

The system of equations is solved with the use of generalized curvilinear coordinates (see [1] for details). It is a corrector-predictor scheme, as proposed by Gottlieb and Turkel [11] for the periodic case, but the finite difference approximation is now replaced by the compact scheme McCormack formulation, second order in time and fourth order in space, devised by Kennedy and Carpenter [12]. It is given by:

$$
\begin{aligned}
& a D_{i-1}^{\mathrm{B}}+(1-a) D_{i}^{\mathrm{B}}=\frac{1}{\Delta x}\left(f_{i}-f_{i-1}\right) \\
& a D_{i+1}^{\mathrm{F}}+(1-a) D_{i}^{\mathrm{F}}=\frac{1}{\Delta x}\left(f_{i+1}-f_{i}\right)
\end{aligned}
$$

where $D$ is the numerical approximation to the spatial derivative of the function $f$ (see [13]). The exponents B and F stand respectively for forward and backward differencing and $a$ is a coefficient taken such that $a=\frac{1}{2}-\frac{1}{2 \sqrt{3}}$ (see [13]). The advantage of the compact scheme is that it is more precise when highly distorted grids are used, thus allowing us to take into account more complex duct geometries. Another advantage is the high performance of its boundary stencils (see [12] and [13]).

As previously pointed out, it is a challenging problem to impose proper boundary conditions for subsonic unsteady flows that exhibit spatial growth. First, to obtain a fully developed turbulent field at the entry of our computational domain, the inlet conditions for the spatially growing duct flow are provided, at each time step, by a periodic non-heated duct that is computed concurrently. The periodic duct boundary conditions at the walls are no-slip and non-heated with an imposed temperature $T_{\mathrm{w}}$. The wall pressure is obtained by solving the Navier-Stokes equations at the wall.

For the spatial duct, the wall boundary conditions are also no-slip and the wall pressure is determined as previously. The spatial duct is linked to the periodic duct by the characteristic boundary conditions proposed by Poinsot and Lele [3] (see also [14]). Both periodic and spatial simulations use the same time step $\operatorname{Min}\left(\Delta t_{\mathrm{p}}, \Delta t_{\mathrm{s}}\right)$ corresponding to the minimum time step found for the periodic and spatial configurations. The velocity and the temperature field computed at the last section of the periodic duct are used to specify the inflow conditions at the first section of the spatial duct (see figure 1). This condition corresponds to a subsonic inflow with fixed velocities and temperature as described by Poinsot and Veynante [14]. For a subsonic flow, four characteristic waves enter the domain with speeds $\lambda_{i}=u, u, u$ and $u+c$ and one of them leaves the domain at the speed $\lambda_{1}=u-c$, see figure 1. $c$ is the speed of sound given for an ideal gas by: $c=(\gamma p / \rho)^{0.5}$, where $p$ and $\rho$ are, respectively, the local instantaneous pressure and the local density at the boundary. $u$ is the local instantaneous flow velocity normal to the boundary.

Since only the velocity components and the temperature can be prescribed, the density $\rho$ (or the pressure $p$ ) has to be determined through a so-called numerical boundary condition (see [14]): the corresponding characteristic wave leaving the domain is therefore determined through the values of the variable at the internal points.

At the outlet, the subsonic partially reflective outflow boundary conditions have been implemented [14]. Similarly to the inflow, four waves leave the domain with speeds $\lambda_{i}=u, u, u$ 


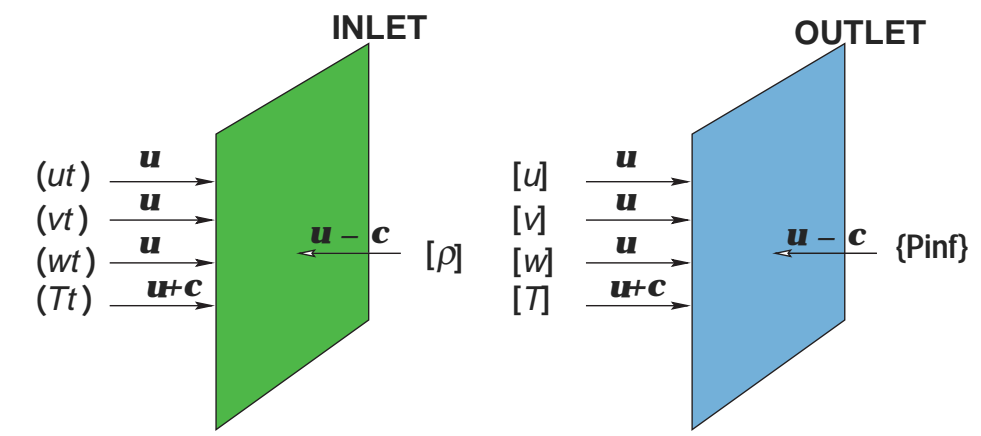

$u$, flow velocity: $c$, sound velocity *: $t$, temporal duct data

() Imposed values

[] Internal points computed values

\{\} Reference value. Soft boundary condition

Figure 1. Inlet and outlet boundary conditions in the spatial duct.

and $u+c$ and one enters the domain at speed $\lambda_{1}=u-c$. The four leaving waves are estimated from the value of the variables at the internal points. The entering waves are computed from a soft boundary condition by specifying a given static pressure $p_{\infty}$ at infinity. As described by Poinsot and Veynante [14], reflected waves enter the domain to bring the mean pressure back to a value close to $p_{\infty}$. A constant $K$ sets the amplitude of the reflected waves [14]. The constant $K$ has an arbitrary value and the number of reflected waves depends of this value. For this work $K=0.99$ [14].

As a validation, we first consider a LES of a non-heated spatial duct with all the four walls at the imposed temperature $T_{\mathrm{w}}$. Figure 2 allows evaluation of the convergence rate of the characteristic waves boundary conditions. Based on the mass conservation, the boundary condition is converged when the value $\left|Q_{\text {out }}-Q_{\text {in }}\right|$ is close to zero. $Q_{\text {in }}$ is the mass flux at the duct inlet $\left(x / D_{\mathrm{h}}=0.0\right)$ and $Q_{\text {out }}$ the corresponding mass flux at the outlet $\left(x / D_{\mathrm{h}}=L_{x}\right) \cdot L_{x}$ stands for the longitudinal length of the duct. The mass flux is defined as:

$$
Q=\int_{0}^{D_{\mathrm{h}}} \int_{0}^{D_{\mathrm{h}}} u(y, z, t) \rho(y, z, t) \mathrm{d} y \mathrm{~d} z
$$

A transitional period is observed until $t \approx 90 D_{\mathrm{h}} / U_{\mathrm{b}}$ with strong fluctuations at first and which are then damped indicating a convergence of the boundary conditions. In this paper, flow structures are identified using the so-called $Q$ criterion $[15,16]$, which is based on the second invariant of the velocity gradient tensor. Positive $Q$ regions correspond to flow regions where the vorticity dominates the deformation and have been shown to be a good indicator of coherent vortices.

A visual method of checking the cleanness of the inlet boundary conditions is given in figure 3 and animation 3 which show that the turbulent structures identified through the positive $Q$ isosurfaces exhibit no discontinuity while crossing the border between the periodic and the spatial duct. We have checked that similar continuity is observed on all the instantaneous variables (velocity, density or temperature).

As compared with the periodic duct for which spatial averaging in the flow direction can be combined with temporal averaging to reach a faster convergence of the statistical quantities, the spatial duct requires a much longer time integration to obtain a statistical convergence. Furthermore, as stressed above, the usable computation starts only when the initial oscillations due to the establishment of correct boundary conditions are damped out. For the present LES, about six times the time period of crossing $T_{\mathrm{C}}=L_{x} / U_{\mathrm{b}}$ is needed for boundary condition 

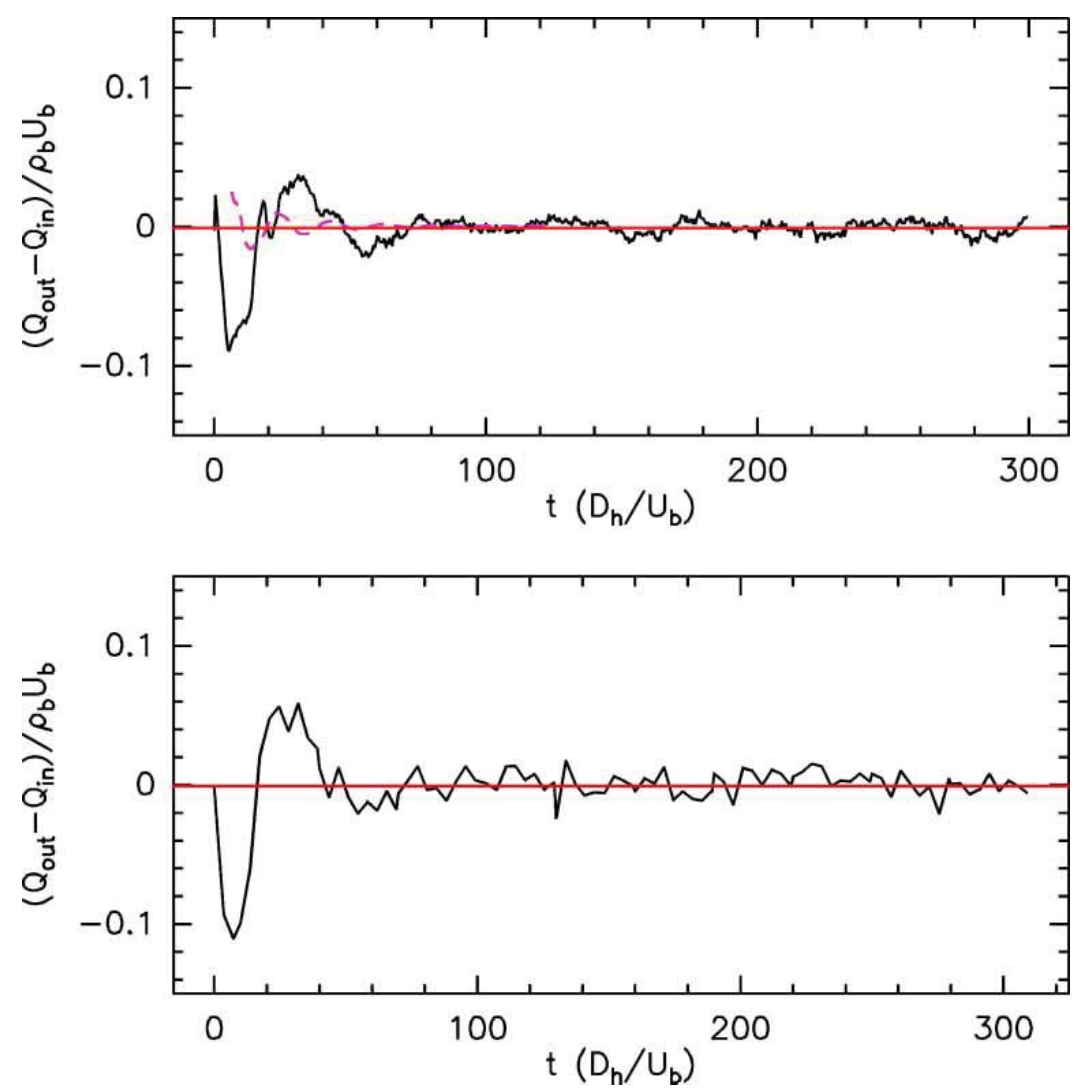

Figure 2. Boundary conditions convergence. The figure shows the difference between the mass flux at the outlet $\left(x / D_{\mathrm{h}}=L_{x}\right), Q_{\text {out }} /\left(U_{\mathrm{b}} \rho_{\mathrm{b}} D_{\mathrm{h}}^{2}\right)$ and the mass flux at the inlet, $Q_{\text {in }} /\left(U_{\mathrm{b}} \rho_{\mathrm{b}} D_{\mathrm{h}}^{2}\right)\left(x / D_{\mathrm{h}}=0\right)$ as a function of the time. The time is non-dimensionalized by $D_{\mathrm{h}} / U_{\mathrm{b}}$. Top, heated ducts (—— LES-temp, --- LES-flux); bottom, non-heated duct ( $(-)$.

convergence and about $14 T_{\mathrm{c}}$ in addition to reach an acceptable statistical convergence. Due to the long time integration required, a relatively low spatial resolution has to be used in the present LES. The size of the computational domain is taken equal to $15 D_{\mathrm{h}} \times D_{\mathrm{h}} \times D_{\mathrm{h}}$ (in the $x$ streamwise, $y$ and $z$ transverse directions, respectively) for the non-heated spatial duct, $31 D_{\mathrm{h}} \times D_{\mathrm{h}} \times D_{\mathrm{h}}$ for both heated spatial ducts and $6.4 D_{\mathrm{h}} \times D_{\mathrm{h}} \times D_{\mathrm{h}}$ for the precursory periodic duct. The computational grid consists of $160 \times 50 \times 50$ nodes in $x, y$ and $z$ for the non-heated spatial duct, $318 \times 50 \times 50$ nodes for both heated ducts and $64 \times 50 \times 50$ nodes in $x, y$ and


Figure 3. Visualization of the crossing of the turbulent structures from the periodic duct to the spatial duct. Positive $Q$ isosurfaces with $Q=0.5\left(U_{\mathrm{b}} / D_{\mathrm{h}}^{2}\right)$. Click here for animation. 
$z$ for the periodic inlet duct. A non-uniform grid with a hyperbolic-tangent stretching is used in the $y$ and $z$ directions for both ducts. Distribution and discretization points are identical in the $y$ and $z$ direction for both ducts. A uniform grid is used in the streamwise direction for the periodic duct as well as for the non-heated spatial duct until $x / D_{\mathrm{h}}=14.6$ and for both heated ducts until $x / D_{\mathrm{h}}=30.5$. Further downstream, a longitudinally refined grid is used to avoid the creation of spurious reflected waves. All simulations are performed at a bulk Reynolds number $R e_{\mathrm{b}}=6000$ and Mach $=0.5$. The Reynolds and Mach numbers are based on the bulk velocity $\left(U_{\mathrm{b}}\right)$, the bulk density $\left(\rho_{\mathrm{b}}\right)$, the hydraulic diameter $\left(D_{\mathrm{h}}\right)$ and the wall temperature $\left(T_{\mathrm{w}}\right)$. To properly determine the grid point distribution close to the walls, one first has to estimate the viscous length. It can be determined a priori through the friction velocity obtained by the Jones equation [17] (see [1]). Based on this estimation, the grid distribution varies from 1.8 to 14 wall units in the $y$ and $z$ directions for both ducts. The grid spacing in the flow direction $x$ is $\Delta x^{+}=48$ for the periodic duct and varies from 48 to 2.5 in the spatial configuration. The Reynolds number based upon the friction velocity is $R e_{\tau}=393$. For all the cases, the heated wall will correspond to $y=0$.

\section{LES of the spatially growing non-heated duct}

We here consider a non-heated duct with all the walls at the given temperature $T_{\mathrm{w}} \cdot U_{\mathrm{b}}$ and $\rho_{\mathrm{b}}$ correspond to the bulk velocity and bulk density of the precursory periodic duct. To check that the inlet field for the spatial duct corresponds to a well-developed turbulent field and to test the compact scheme formulation, we first consider a non-heated periodic duct. The results are compared with the DNS results of Gavrilakis [18] in figure 4. Despite the low resolution of the LES computation, a very good agreement is observed between the DNS and the LES for both first and second order statistics. As far as the non-heated spatial duct is concerned, a perfect prescription of the outlet boundary conditions (that is to say the pressure at infinity) should yield a flow statistically identical to the flow in the non-heated periodic duct: the statistics should therefore be independent of the streamwise direction.

Due to the slightly incorrect prescription of $p_{\infty}$, we observe a slight difference between the mass flux in the periodic duct and that of the spatial duct. The mass flux goes indeed from $\mathrm{Q}=$ $1 \rho_{\mathrm{b}} U_{\mathrm{b}} D_{\mathrm{h}}^{2}$ in the periodic duct to $\mathrm{Q} \approx 1.07 \rho_{\mathrm{b}} U_{\mathrm{b}} D_{\mathrm{h}}^{2}$ in the spatial duct: the bulk velocity shows an increase of $\approx 5 \%$ between the two ducts. The most strongly affected variable is the friction velocity, for which the differences reach $\approx 12 \%$ due to the simultaneous changes in velocity and density close to the walls. This slight discrepancy could be solved by trying several outlet pressures $p_{\infty}$, but these tests are very consuming as far as CPU time is concerned since the boundary conditions must have converged in order to know the precise mass flux in the spatial duct.

Despite this change in the flow mass flux, the normalized statistical quantities at various downstream locations of the spatial duct are almost identical to those measured in the periodic duct in [1]. Figure 5 compares the mean profiles of various quantities as a function of the distance $y$ from the wall at a fixed distance $z / D_{\mathrm{h}}=0.5$ corresponding to the centre line of the square duct. The profiles are computed at six different $x$ locations for the spatial duct when streamwise averaging is performed in the periodic case. Very good agreement between the spatial duct and the periodic duct measurements is observed for the mean transversal velocity profiles (mean secondary flow) as well as for the various second order velocity statistics. It is important to note that the profiles vary only very slightly as a function of the $x$ location, showing that the implemented boundary conditions allow for a good reproduction of the streamwise flow statistical homogeneity. A variable that is very sensitive to the quality of the computation is the wall shear stress plotted in figure 6. Similar to the other statistical 
(a)

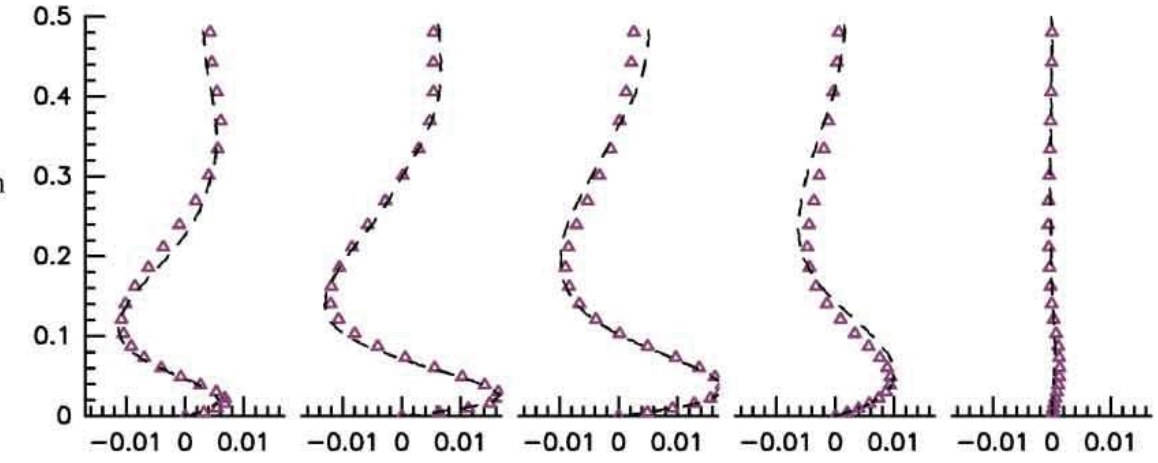

$W / U_{\mathrm{b}}$

(b)

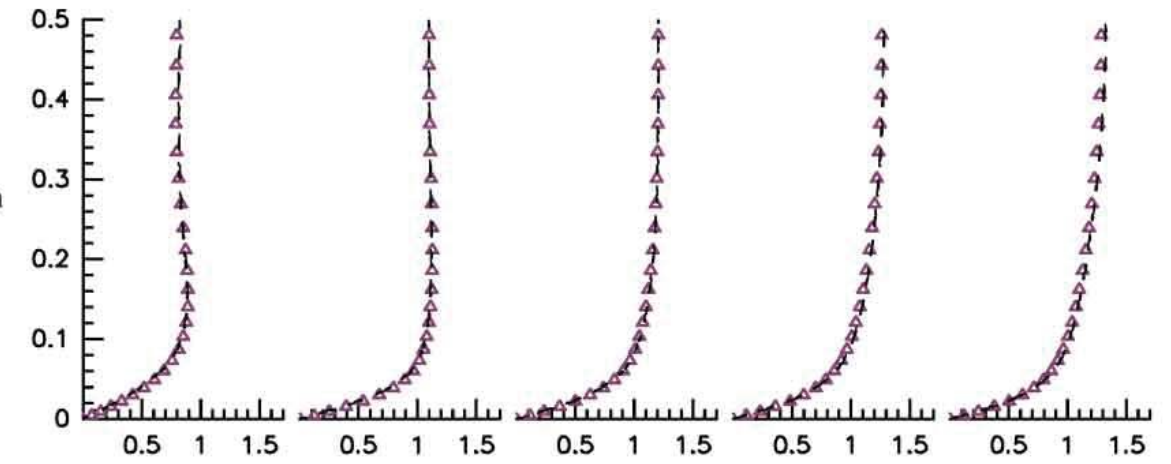

(c)

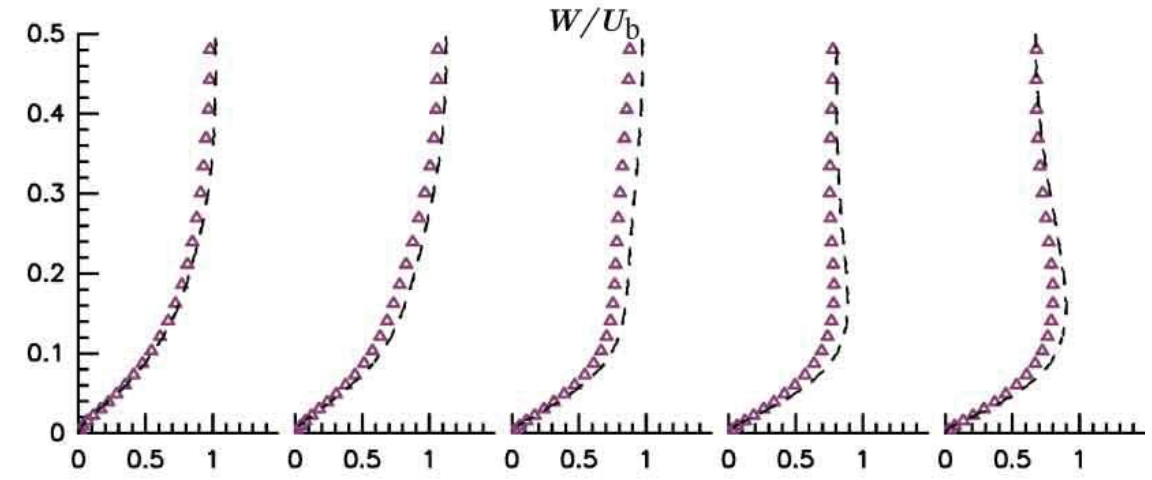

$u_{r m s} / U_{\tau}$

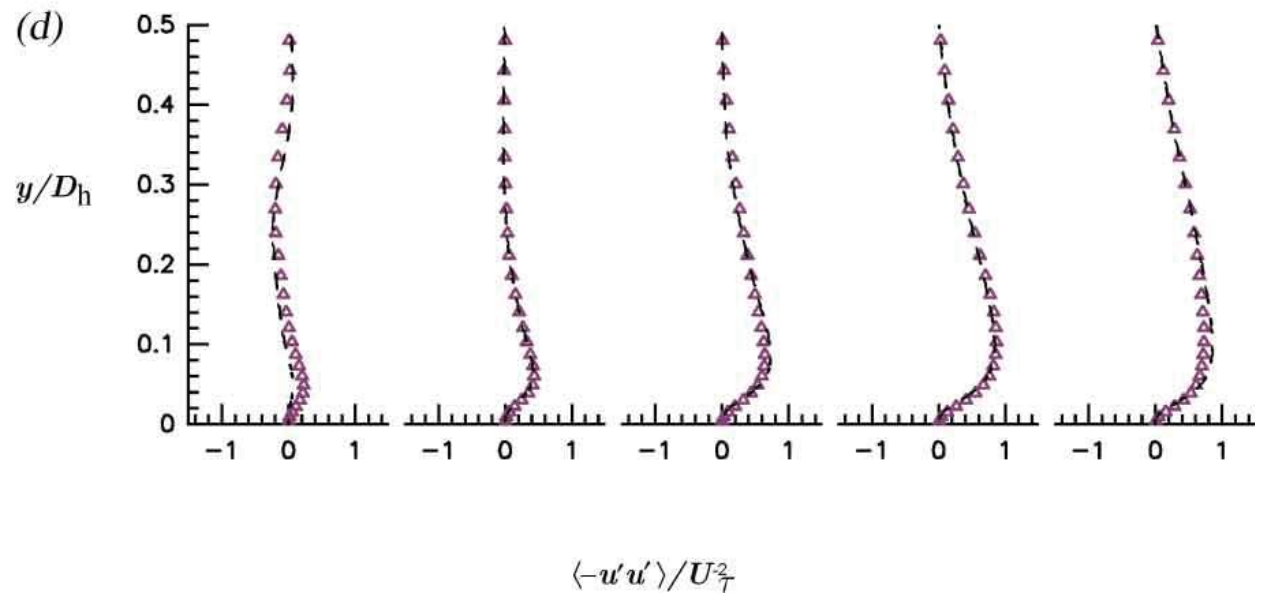

Figure 4. Periodic non-heated duct. Comparison between the results of the present LES $(\triangle)$ and Gavrilakis' DNS [18] (--) for five different lateral locations $z / D_{\mathrm{h}}=0.05,0.15,0.25,0.35$ and 0.5 . Values are normalized by the bulk velocity $\left(U_{\mathrm{b}}\right)$ or the friction velocity $\left(U_{\tau}\right)$. 

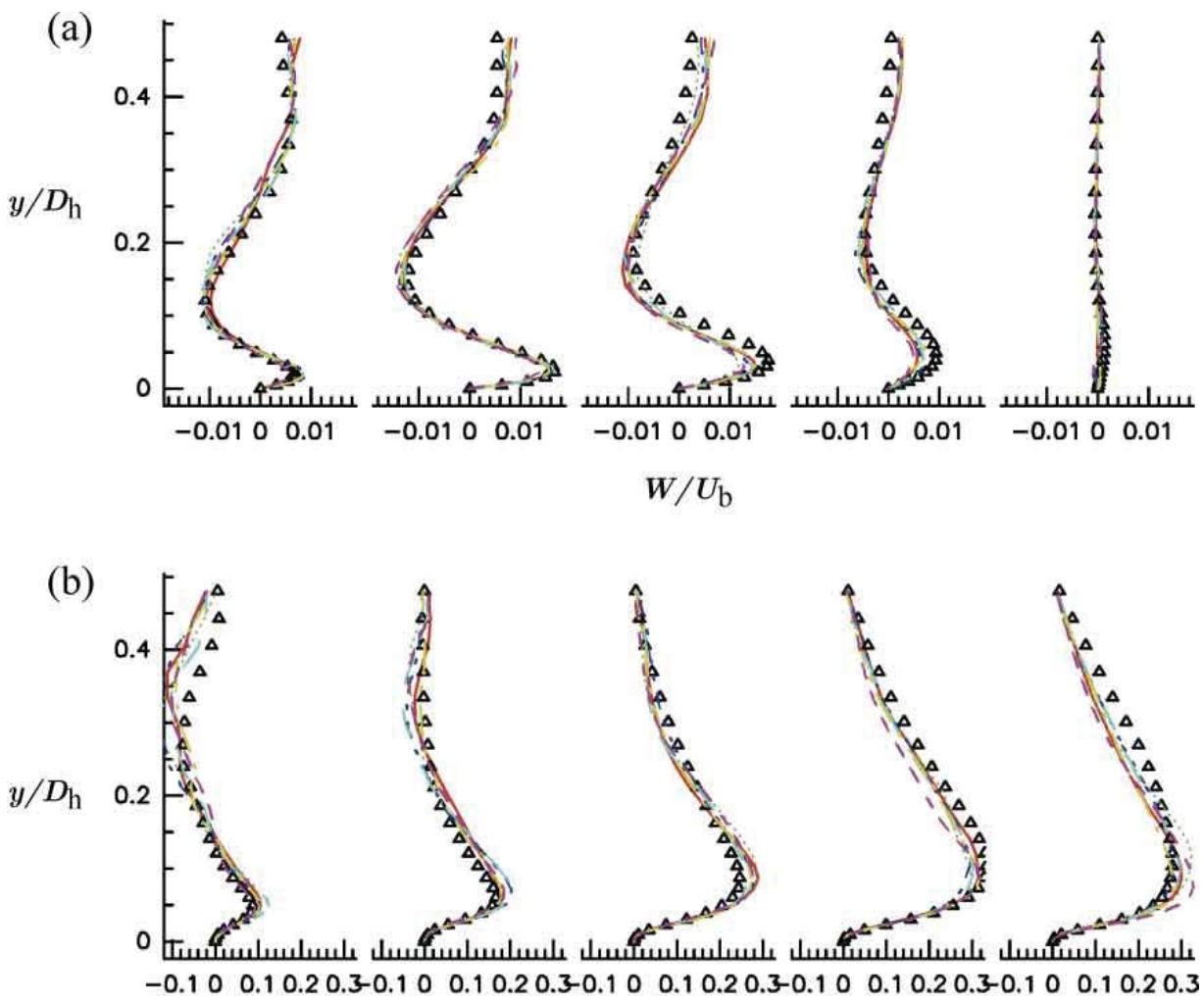

$100\left\langle-u^{\prime} u^{\prime}\right\rangle / \boldsymbol{U}_{\mathrm{b}}^{2}$
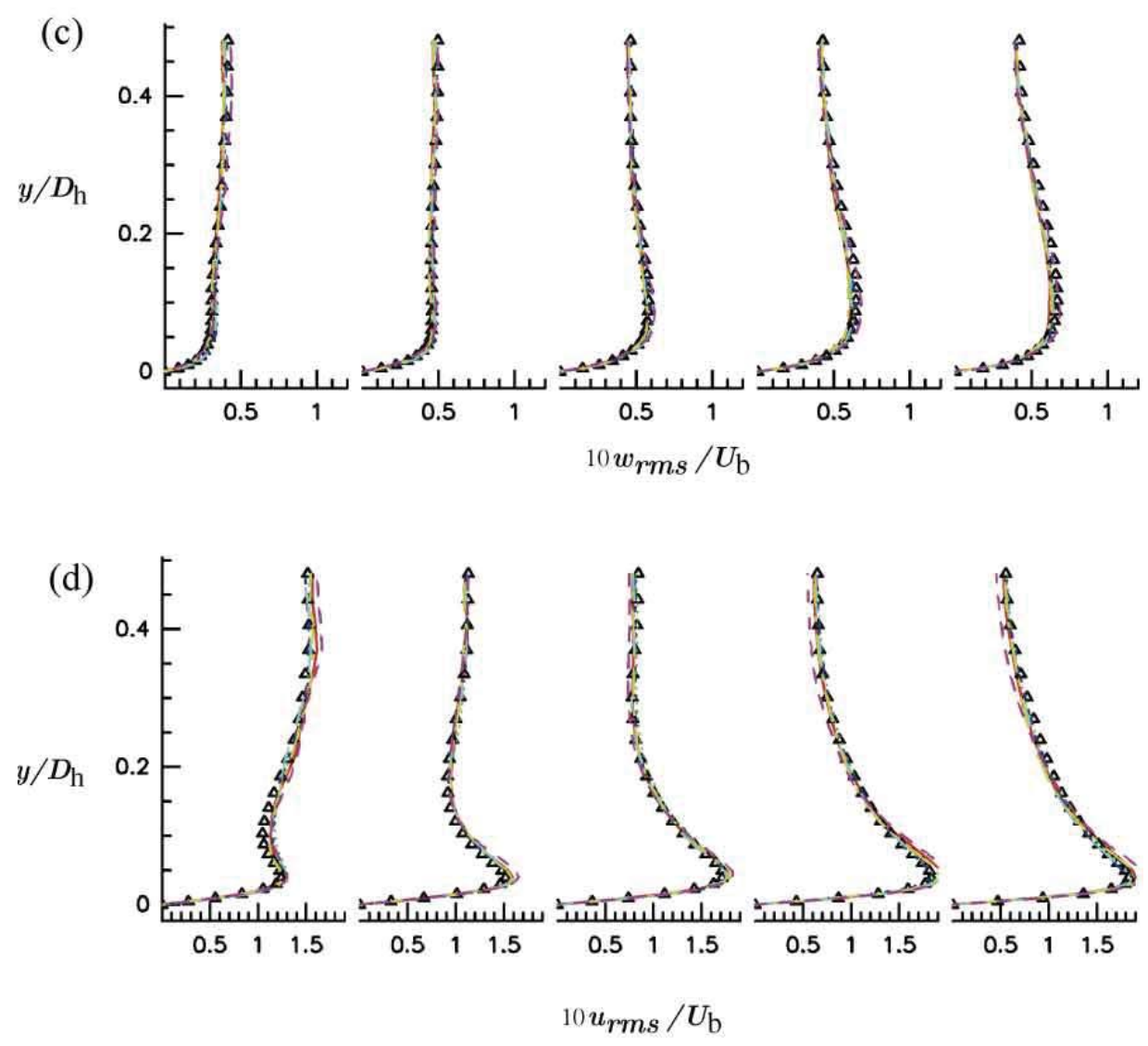

Figure 5. Non-heated spatial duct statistics at five different $x$-planes. Profiles as a function of the distance $y$ from the wall and at five different fixed distance $z / D_{\mathrm{h}}=0.05,0.15,0.25,0.35$ and 0.5 from the lateral wall. $x / D_{\mathrm{h}}=1.0$, - - -; $x / D_{\mathrm{h}}=3.0, \ldots . . ; x / D_{\mathrm{h}}=7.0,-\longrightarrow ; x / D_{\mathrm{h}}=10.0,-. \cdot-; x / D_{\mathrm{h}}=12.0, \longrightarrow ; x / D_{\mathrm{h}}=15.2,---;$ periodic duct results, $\triangle$. The values are normalized by the local bulk velocity. 


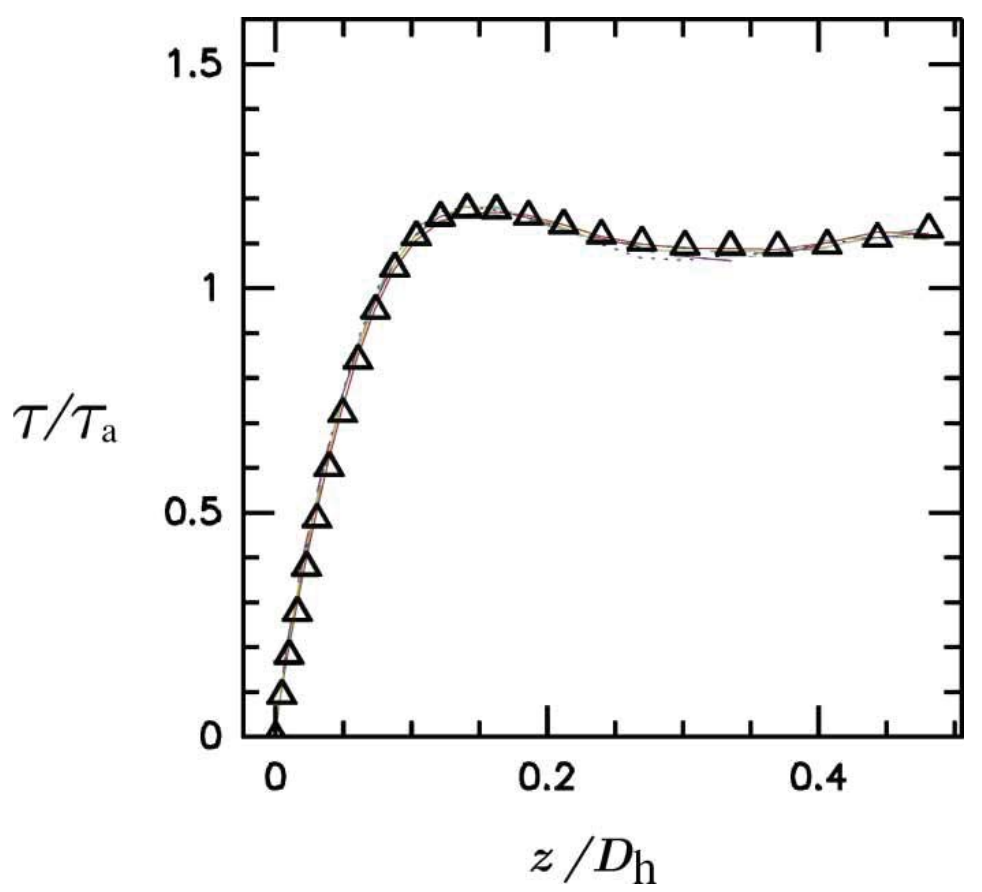

Figure 6. Local wall shear stress $(\tau)$ normalized by the local averaged wall shear stress $\left(\tau_{\mathrm{a}}\right)$ as a function of the distance from the lateral wall $z . x / D_{\mathrm{h}}=1.0,---; x / D_{\mathrm{h}}=3.0, \ldots \ldots ; x / D_{\mathrm{h}}=7.0,--; x / D_{\mathrm{h}}=10.0,-\cdot-\cdot$; $x / D_{\mathrm{h}}=12.0, \ldots ; x / D_{\mathrm{h}}=15.2,---$; periodic duct results [1], $\triangle$.

quantities, the normalized shear stress profile as a function of the distance $z$ from the lateral wall compares very well with the periodic results obtained in [1] and is identical for all the considered downstream locations.

\section{LES of spatially growing heated ducts}

Two distinct LESs of spatial heated ducts are performed, differing in the way wall heating is applied: in the first LES, the temperature of one of the four walls is abruptly changed from $T_{\mathrm{w}}$ to $T_{\mathrm{h}}=2.5 T_{\mathrm{w}}$ for $x / D_{\mathrm{h}} \geq 0.4$. The other three walls have a constant temperature $T_{\mathrm{w}}$ over the whole length of the duct. Some of the results corresponding to this first configuration have been presented in [2]. This will be referred to as LES-temp. The second LES, referred to as LES-flux, corresponds to heating with a constant heat flux associated with a Nusselt number

$$
N u=H_{\mathrm{w}} /\left(k\left(T_{\mathrm{w}}\right) T_{w} / D_{\mathrm{h}}\right)
$$

equal to 30. $H_{\mathrm{w}}$ is the imposed heat flux $H_{\mathrm{w}}(x, z)=\left.\kappa(T) \frac{\partial\langle T(x, y, z)\rangle}{\partial y}\right|_{y / D_{\mathrm{h}}=0}$ at the wall and $\kappa\left(T_{\mathrm{w}}\right)$ is the fluid diffusivity at temperature $T_{\mathrm{w}}$ determined through the Sutherland law. Heating is also applied for $x / D_{\mathrm{h}} \geq 0.4$. As pointed out in the Introduction, the LES-flux simulation is in closer correspondence with numerous industrial situations. In both cases, the inflow conditions correspond to a fully developed turbulent field issued from a non-heated periodic duct computation. The results are compared with the periodic duct LES conducted by SalinasVázquez and Métais [1]. The parameters of this previous LES are in close correspondence with LES-temp and LES-flux simulations with a bulk Reynolds number $R e_{\mathrm{b}}=6000$ and a Mach number Mach $=0.5$. In this periodic simulation, a constant temperature was imposed at the walls with a ratio $T_{\mathrm{h}} / T_{\mathrm{w}}=2.5$ between the temperature of the heated wall and the temperature of the non-heated walls.

As pointed out in [1], the heating yields a strong amplification of the secondary flow in the vicinity of the heated wall. At variance with the periodic case investigated by Salinas-Vázquez 

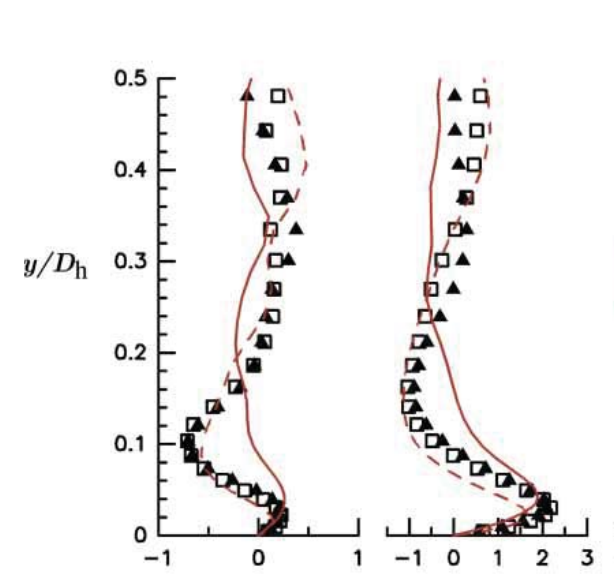

$x / D_{\mathrm{h}}=15$
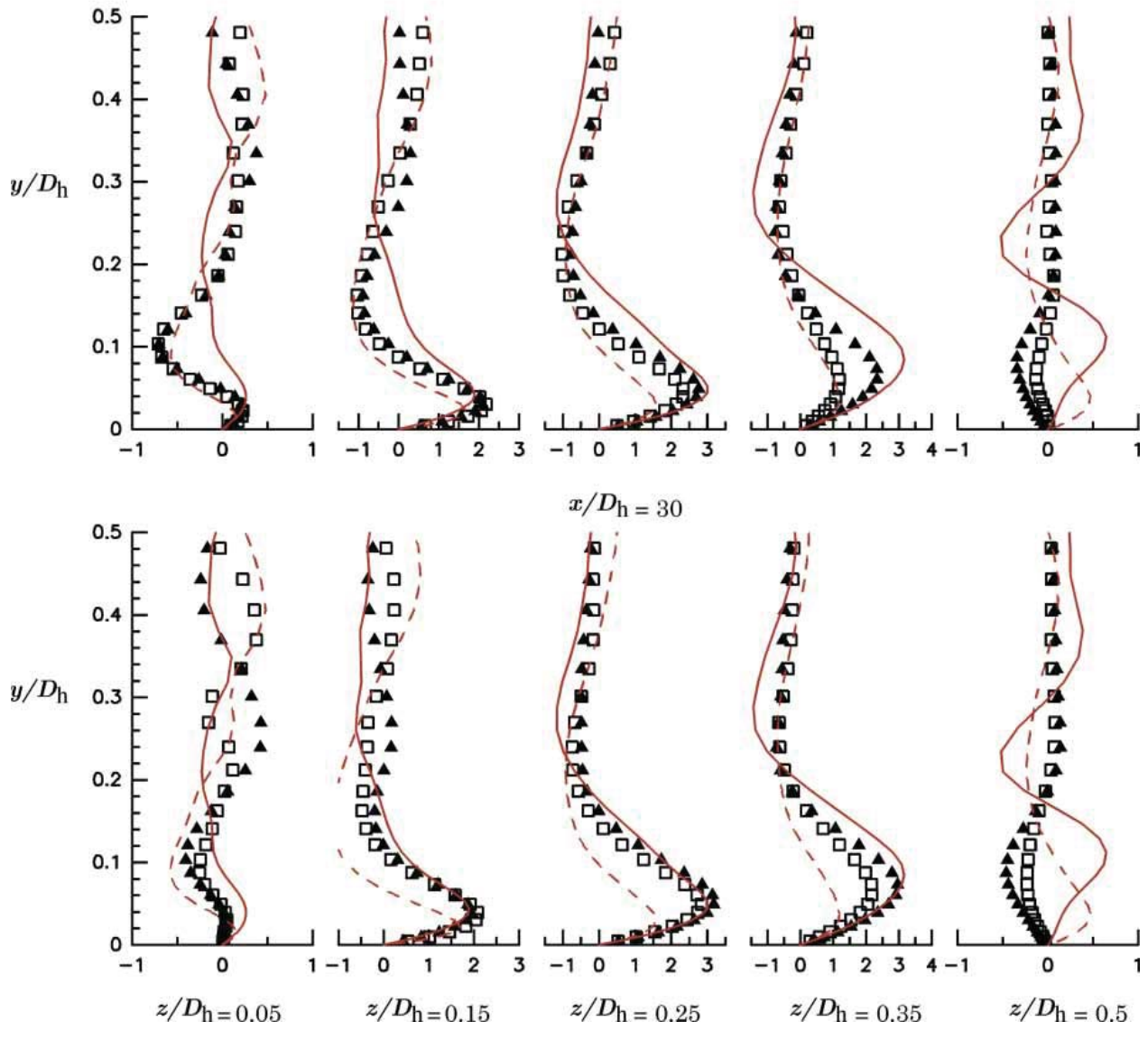

$x / D_{\mathrm{h}}=30$

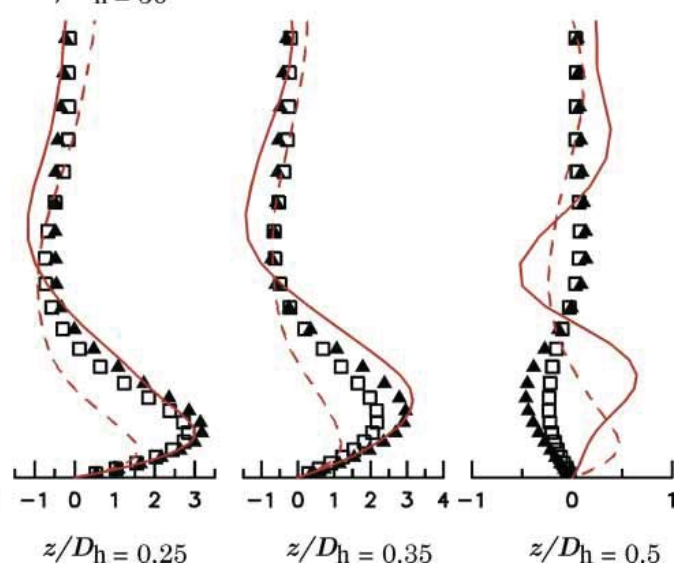

Figure 7. Zoom on the $\langle W\rangle / U_{\mathrm{b}}$ profiles at five $z$-planes: $z / D_{\mathrm{h}}=0.05,0.15,0.25,0.35$ and 0.5; LES-temp, $\mathbf{\Lambda}$; LES-flux, $\square$; periodic heated duct [1], —; non-heated duct, - --.

and Métais [1], which corresponds to a fully developed turbulent and thermal regime, the two spatial configurations see a gradual increase of the thermal boundary layer with downstream distance leading to a progressive modification of the turbulent field as we move downstream. This is illustrated in figure 7 which shows the profiles for the transverse mean velocity profile $W$.

A comparison of LES-temp and LES-flux simulations with the non-heated and heated periodic case indicates progressive displacement of the secondary vortices from the corner toward the duct centre as well as their progressive growth, both in size and intensity, as we move downstream. Near the duct corner $\left(z / D_{\mathrm{h}}=0.05\right)$, we indeed see an important reduction of the transverse velocity between both stations $x / D_{\mathrm{h}}=15$ and $x / D_{\mathrm{h}}=30$, indicating a shift of the vortices towards the duct middle plane. Conversely, at $z / D_{\mathrm{h}}=0.25$ and $z / D_{\mathrm{h}}=0.35$, the secondary flow is amplified and its positive extremum shifts away from the heated wall. As will be confirmed through examination of the thermal variables, the LES-flux simulation sees a progressive increase of the heated wall temperature from $T_{\mathrm{h}}$ near the inflow to $3.6 T_{\mathrm{h}}$ at the duct outlet. The amplification of the secondary flow is therefore much more gradual than in the LES-temp simulation and the transverse flow remains lower even near the duct exit. Figure 7 also shows that the secondary flow is of comparable intensity for the spatially growing cases and for the periodic heated case. However, the vertical extension of the vortex is much more pronounced in the periodic case: this is particularly clear for the profile at $z / D_{\mathrm{h}}=0.35$ which exhibits a negative extremum for $W$ at a greater distance from the heated wall.

This is confirmed in figure 8 which shows the mean velocity field projected on constant- $x$ sections. The inflow conditions for both LES-temp and LES-flux correspond to a non-heated 
(a)
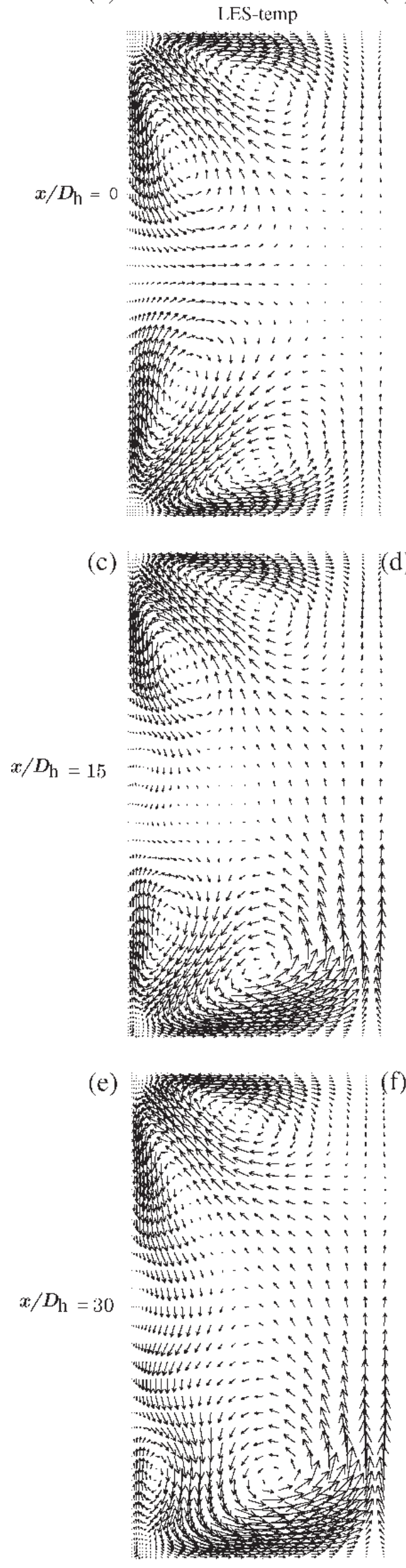

(b)

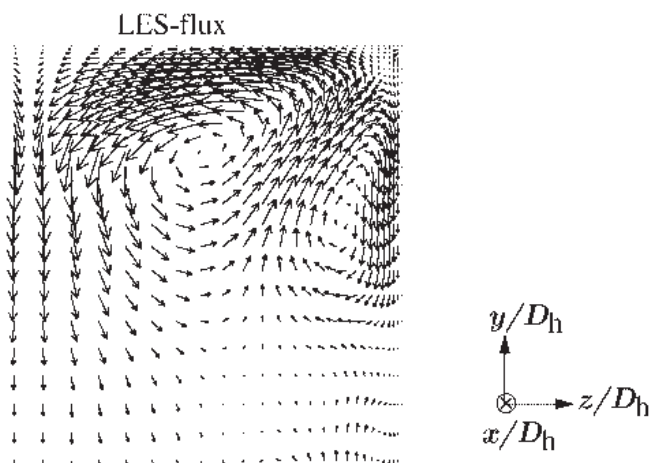

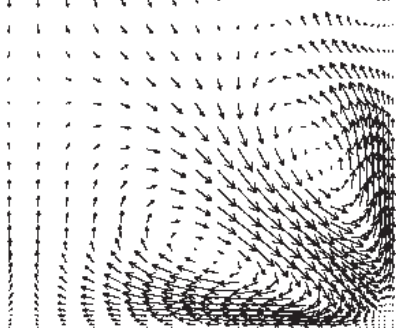

d)

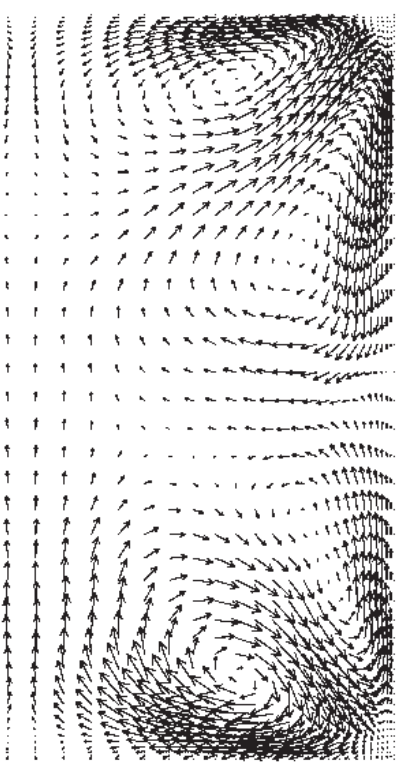

Periodic heated duct

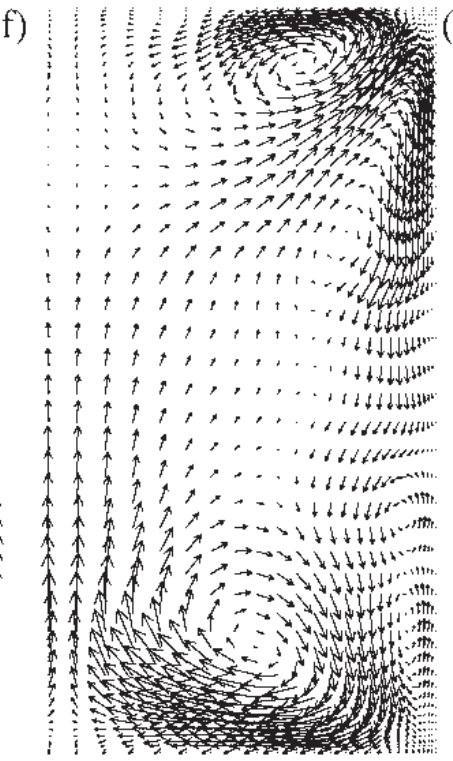



Figure 8. Heated duct mean secondary flows for three $x$-planes, $x / D_{\mathrm{h}}=0,15$ and $=30$. 
(a) $u^{\prime} / U_{\mathrm{b}}$

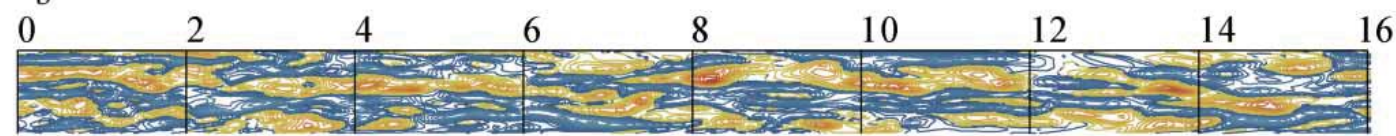

LES-tem
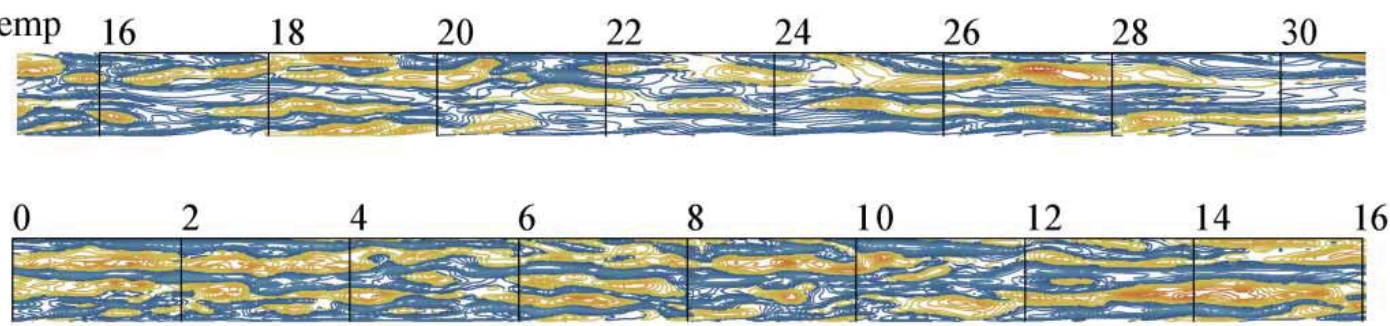

LES-flux

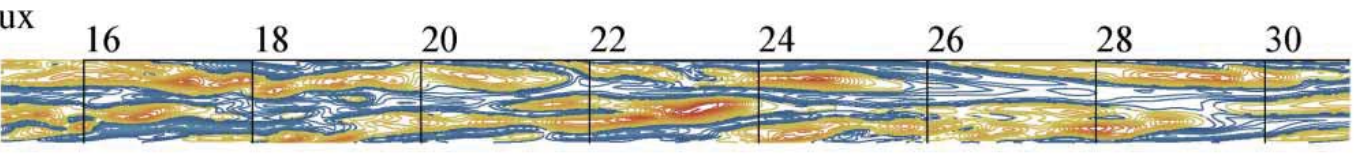

(b) $T^{\prime} / T_{\mathrm{W}}$



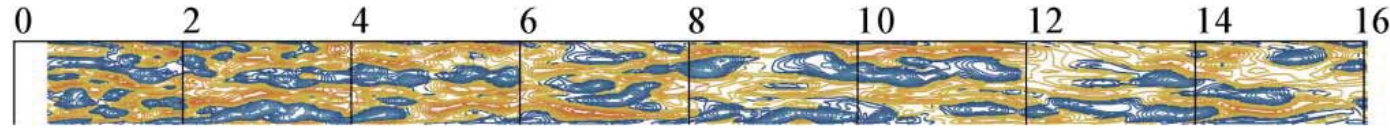

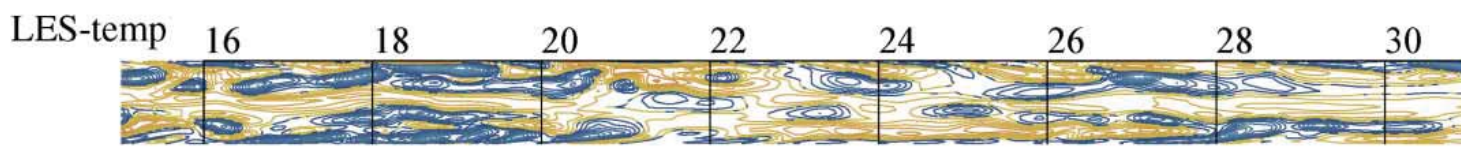

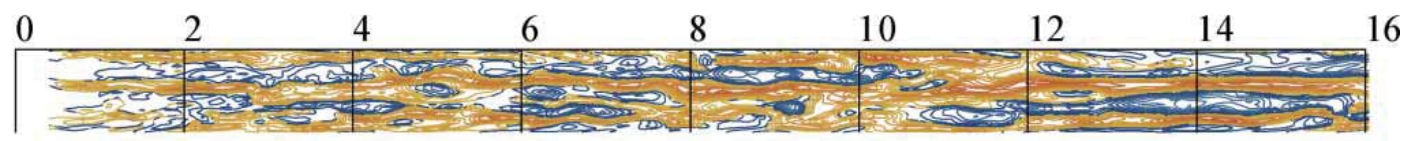

LES-flux

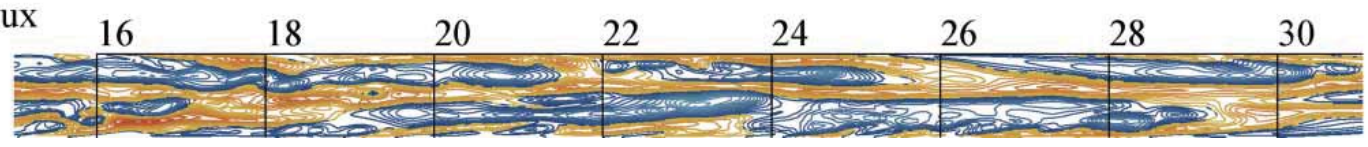

Figure 9. (a) Fluctuating streamwise velocity near the hot wall. (b) Fluctuating temperature near the hot wall.
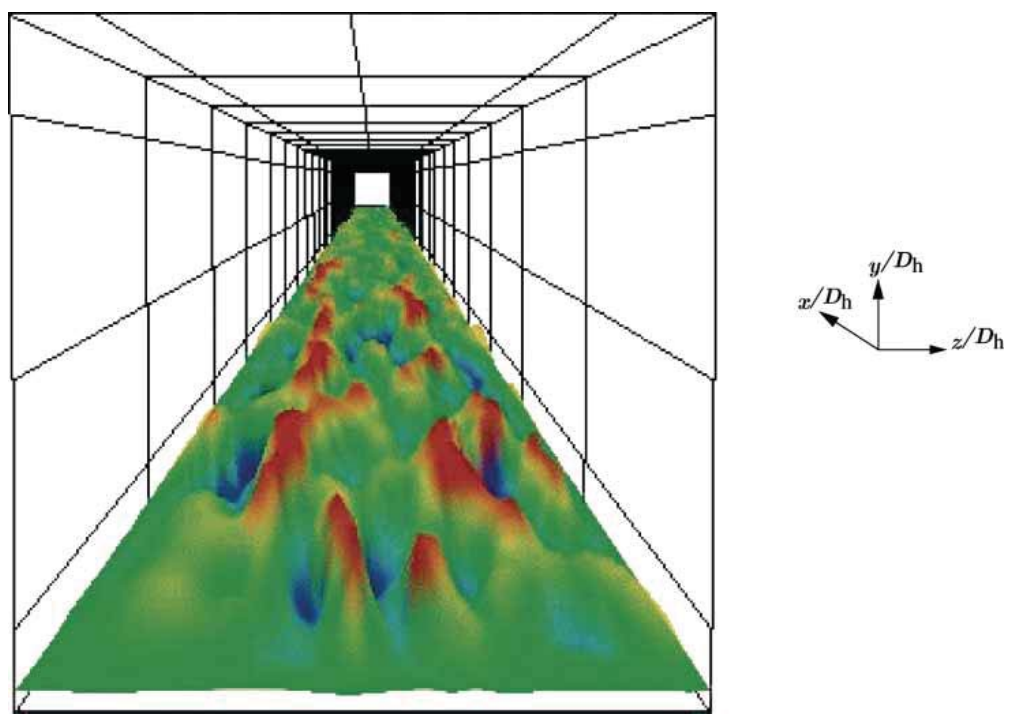

Figure 10. Fluctuating temperature near the hot wall for LES-temp configuration. Click here for animation. 
duct for which counter-rotating pairs of secondary vortices are present near the four corners of the duct. As we go further downstream, the size of the two recirculating vortices near the heated wall is gradually increased and their companion near the adjacent vertical wall is gradually reduced (see also [2]). By the end of the duct, the two vortices are very close together and generate a very strong flow normal to the heated wall. These have a slightly larger extension for LES-temp than for LES-flux. In the periodic case, these vortices are more developed than for the two spatially growing cases. On the wall opposite the heated wall, the pair of two counter-rotating vortices is clearly modified with an enhancement of the size of the vortex adjacent to the lateral wall. This modification is not so apparent for LES-temp and LES-flux.

An important result obtained by Salinas-Vázquez and Métais [1] is related to the viscosity increase near the heated wall associated with the temperature increase. This viscosity enhancement induces an augmentation in size of the characteristic turbulent structures of the

(a)

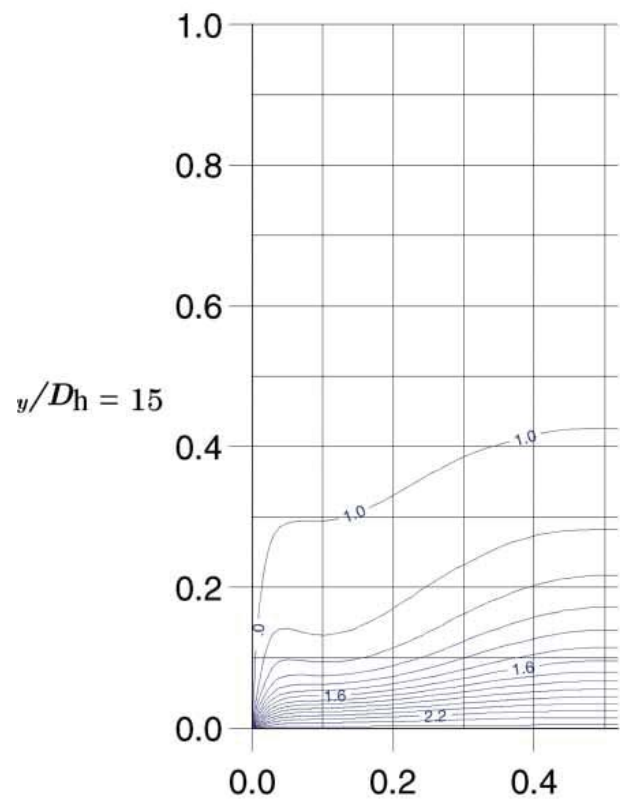

(c)

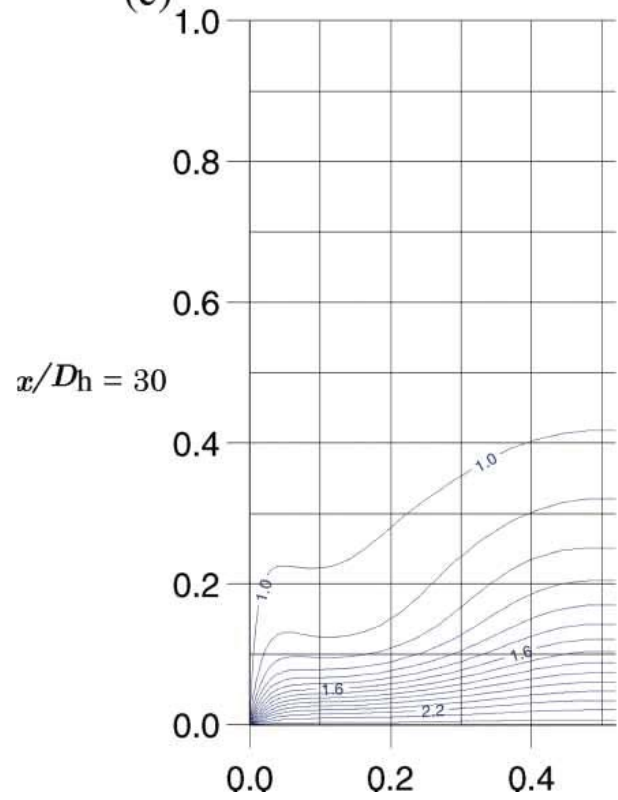

(b) LES-flux
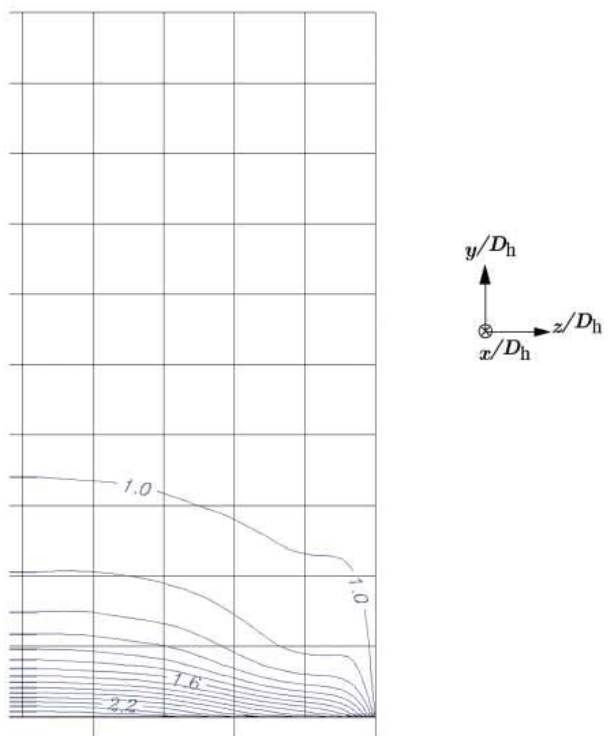

0.6

0.8

1.0

(e)
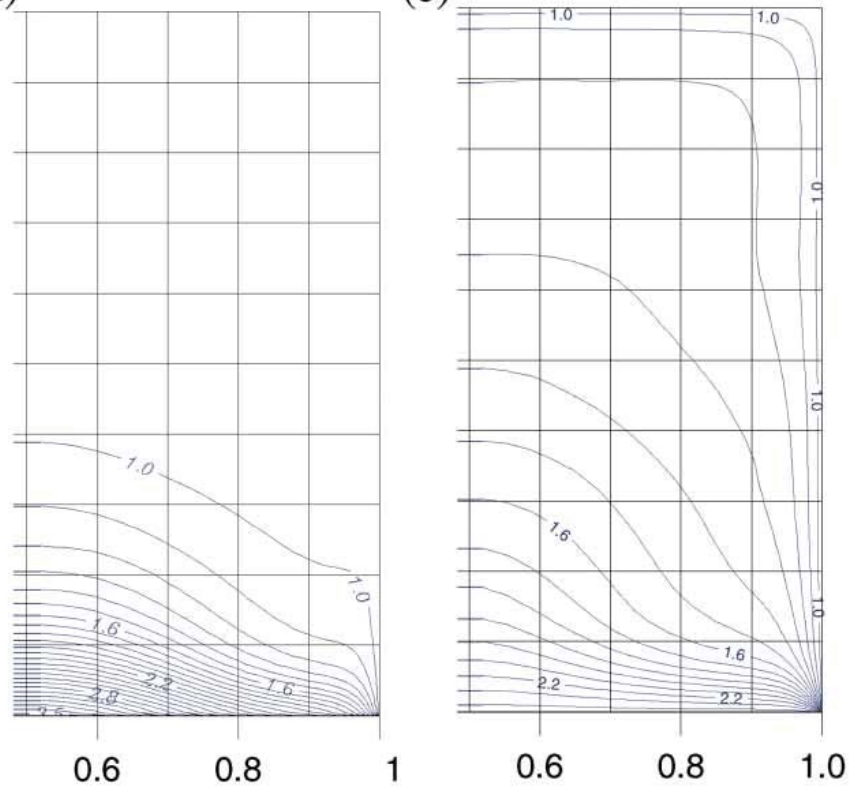

Figure 11. Mean temperature contours, $\langle T\rangle / T_{\mathrm{w}}$, for two different $x$-planes (step $0.1 T_{\mathrm{w}}$ ). 
boundary layer leaving a unique ejection near the middle plane of the heated wall associated with slow and hot fluid expelled from the heated wall (low-speed streak).

In the spatial configuration, we are able to observe the progressive flow transformation due to the growth of the thermal boundary layer. As already observed [2], the streaky structures lengthen and widen as we move in the downstream direction until only one low-speed streak remains, close to the middle of the hot wall. It is then bordered by two high-speed streaks. Figure 9 shows the fluctuating fields for the temperature and the streamwise velocity close the heated wall, in a plane located at $y / D_{\mathrm{h}}=0.01$, for LES-temp and LES-flux. Figure 10 completes the animation for LES-temp. The red colour corresponds to positive values of the fluctuating fields and the blue colour to negative ones. The figure shows that the streaks found close to the end of the duct are quasi-steady indicating that their motion is confined by the limited lateral expansion of the duct.The downstream evolution of the turbulent structures is very similar in both LES-temp and LES-flux cases.

Figure 11 shows the mean temperature contours at two different downstream sections of the duct for LES-temp and LES-flux. The periodic heated case is also added for comparison. The thermal boundary layer is far less developed in the two spatially growing cases, indicating that a very long duct is indeed required to reach the fully developed thermal state corresponding to the periodic case.

This is confirmed by comparing figure 12, which corresponds to the periodic LES [1], and figure 13 obtained in the LES-temp and LES-flux cases. Both figures 12 and 13 show isocontours in a $y-z$ cross-section of the duct of the instantaneous temperature and the fluctuating temperature. For figure 12 , the instantaneous streamwise velocity component $u(\mathbf{x}, t) / U_{\mathrm{b}}$ and the instantaneous fluctuations of the streamwise velocity component $u^{\prime}(\mathbf{x}, t) / U_{\mathrm{b}}$ are shown for comparison. For figure 13, the section is chosen located near the end of the duct at $x / D_{\mathrm{h}}=30$. The red colour indicates maximal values and blue indicates minimal values. Yellow and green are in between. The appearance of an ejection of hot fluid above the heated wall is clearly shown in all the figures. This violent ejection can be observed through the temperature field

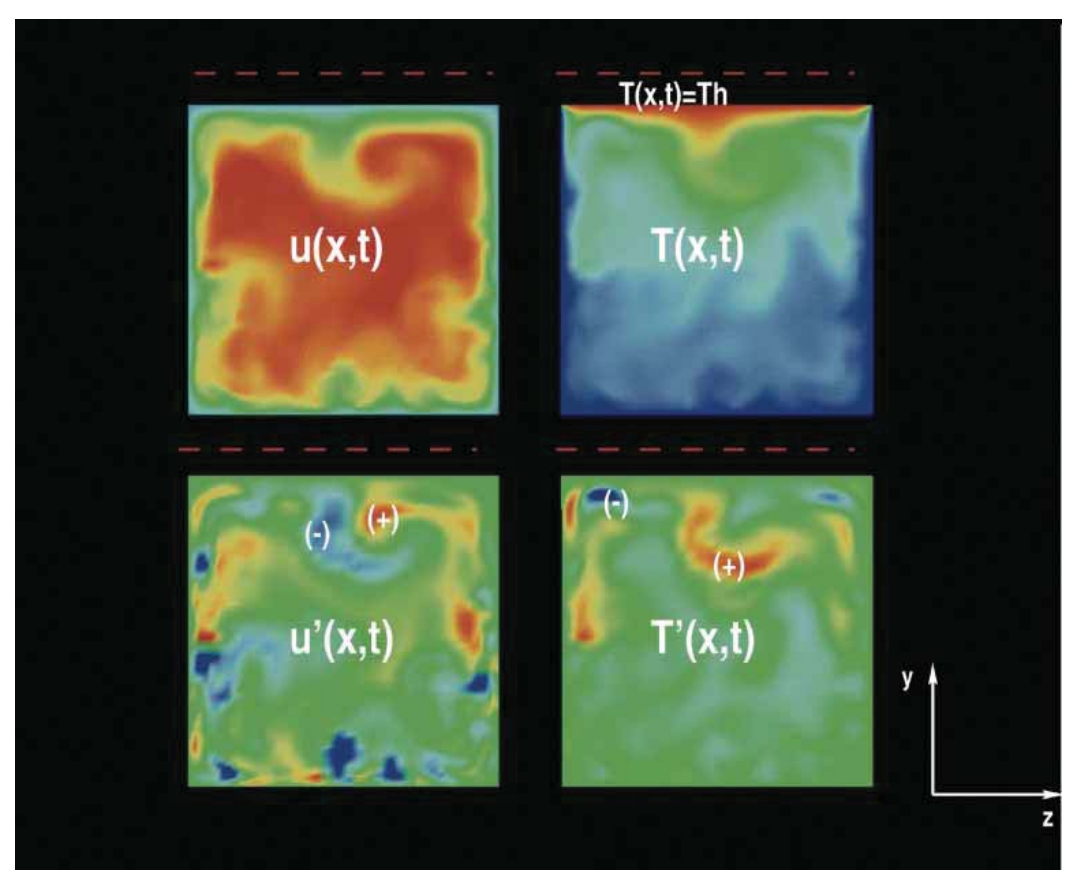

Figure 12. Instantaneous and fluctuating fields for the periodic LES performed by Salinas-Vázquez and Métais [1]. Left: instantaneous, $u(\mathbf{x}, t) / U_{\mathrm{b}}$, (top) and fluctuating, $u^{\prime}(\mathbf{x}, t) / U_{\mathrm{b}}$, (bottom) longitudinal velocity. Right: instantaneous, $T(\mathbf{x}, t) / T_{\mathrm{w}}$, (top) and fluctuating, $T^{\prime}(\mathbf{x}, t) / T_{\mathrm{W}}$, (bottom) temperature. For the instantaneous fields: red, maximal values; blue, minimal values. For the fluctuating field: red, positive values; blue, negative values. 
(a)

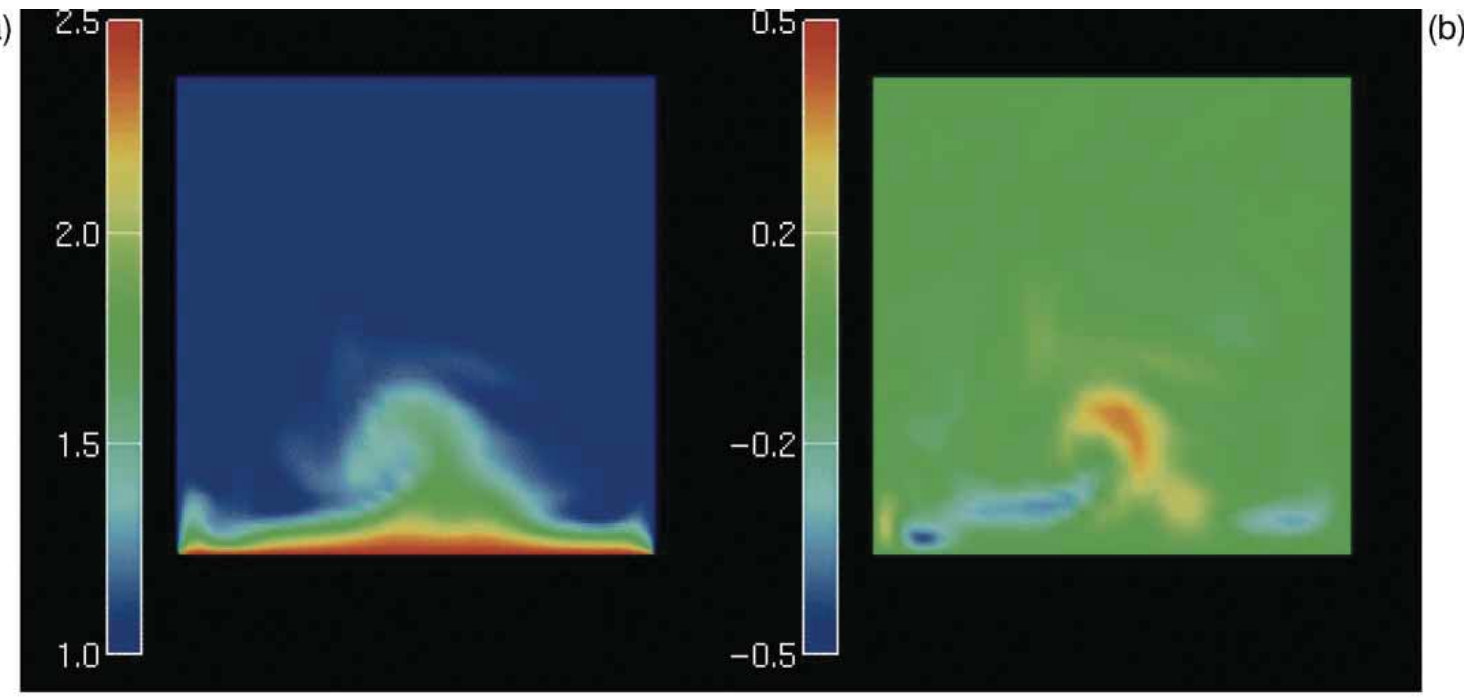

(c)

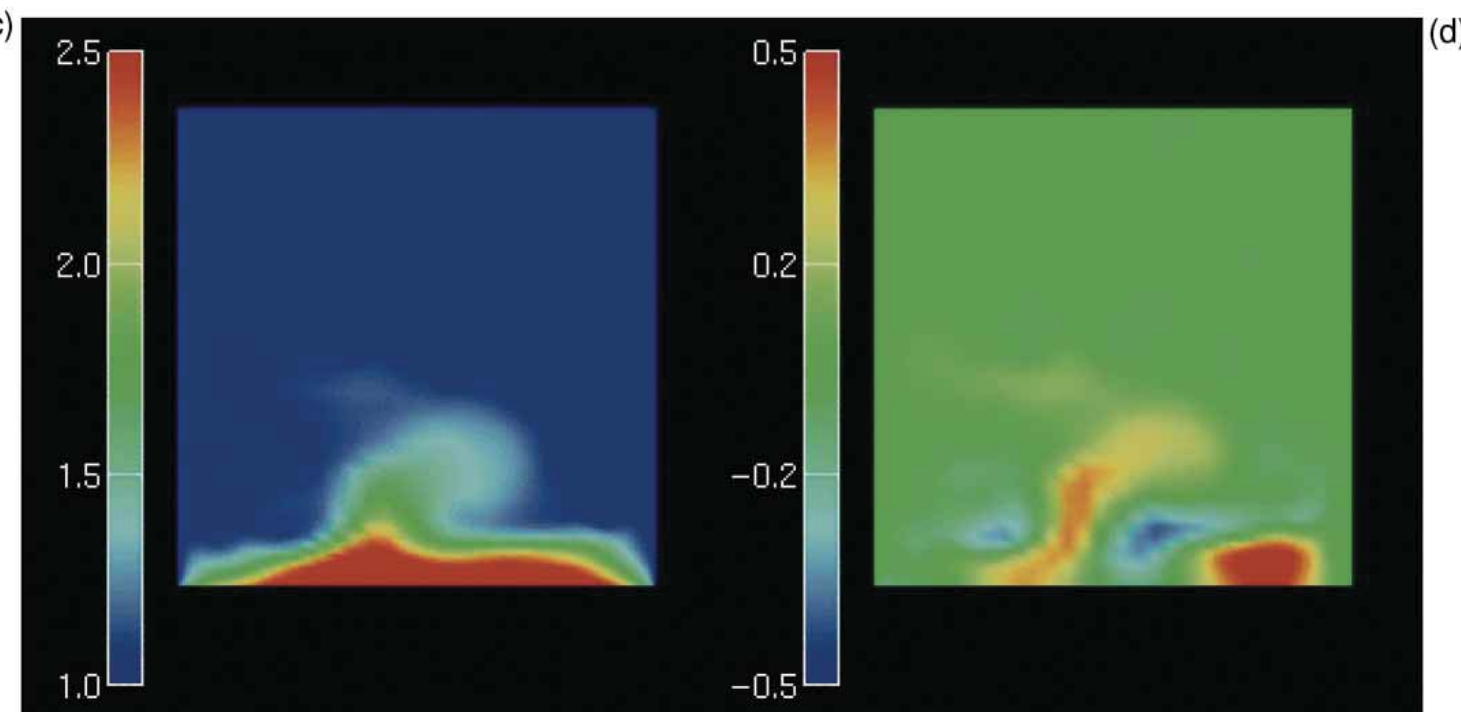

Figure 13. Instantaneous (left) and fluctuating (right) temperature at $x / D_{\mathrm{h}}=30$. For the instantaneous fields: red, maximal values; blue, minimal values. For the fluctuating fields: red, positive values; blue, negative values. Click here for animation (a), (b), (c), (d).

(total and fluctuations) and also through the longitudinal velocity component (figure 12). Indeed, the instantaneous temperature field shows (in red) the expulsion of hot fluid from the heated wall. This region corresponds to a low-speed region of slow fluid coming away from the wall (blue-green). This large-scale structure generates pockets of high-amplitude fluctuating temperature and longitudinal velocity. The ejection is associated with $T^{\prime}(\boldsymbol{x}, t) / T_{\mathrm{w}}>0$ (red) and $u^{\prime}(\boldsymbol{x}, t) / U_{\mathrm{b}}<0$ (blue) zones since it transports hot and slow fluid away from the wall. Around it, the engulfment of cold and high-speed fluid from the core duct (sweeps) towards the wall create regions with $T^{\prime}(\vec{x}, t) / T_{\mathrm{w}}<0$ (blue) and $u^{\prime}(\vec{x}, t) / U_{\mathrm{b}}>0$ (yellow-red). It is clear, when comparing figures 12 and 13, that the extension towards the duct core of the hot fluid ejection is much reduced for the two spatially growing simulations LES-temp and LES-flux.

Going back to figure 11, the gradual increase of the wall temperature for LES-flux yields a much slower growth of the thermal boundary layer than for LES-temp: at $x / D_{\mathrm{h}}=15$, the boundary layer extension is indeed much less pronounced for LES-flux. It becomes comparable near the duct end at $x / D_{\mathrm{h}}=30$. It is important to note that the presence of the secondary flow is associated with very large variations of the thermal properties along the transverse direction of the heated wall. 


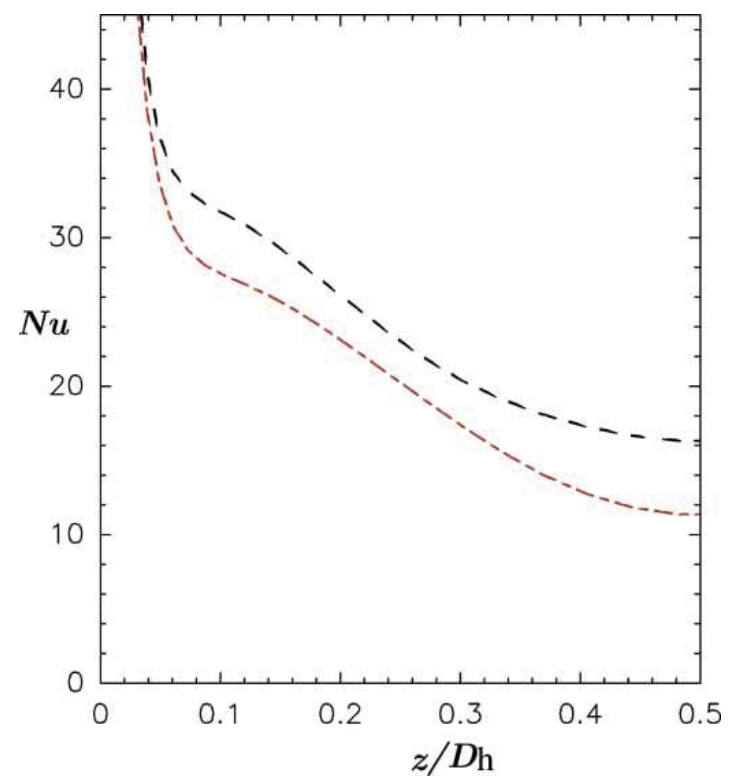

Figure 14. Profiles of the wall heat flux at the heated wall, LES-temp case: $x / D_{\mathrm{h}}=15,--; x / D_{\mathrm{h}}=30,-\cdot-$.

Let us first consider the LES-temp case. Figure 14 shows the profiles of the mean wall heat flux at two downstream locations $x / D_{\mathrm{h}}=15$ and $x / D_{\mathrm{h}}=30$ as a function of the distance to the duct corner. The wall heat flux is defined through the Nusselt number (see equation (18)). Since the wall heat flux is proportional to the mean temperature gradient at the wall, the ejection in the central plane is associated with a minimal value of the flux. At this location, the hot fluid from the heated wall is indeed transported towards the duct core, generating a

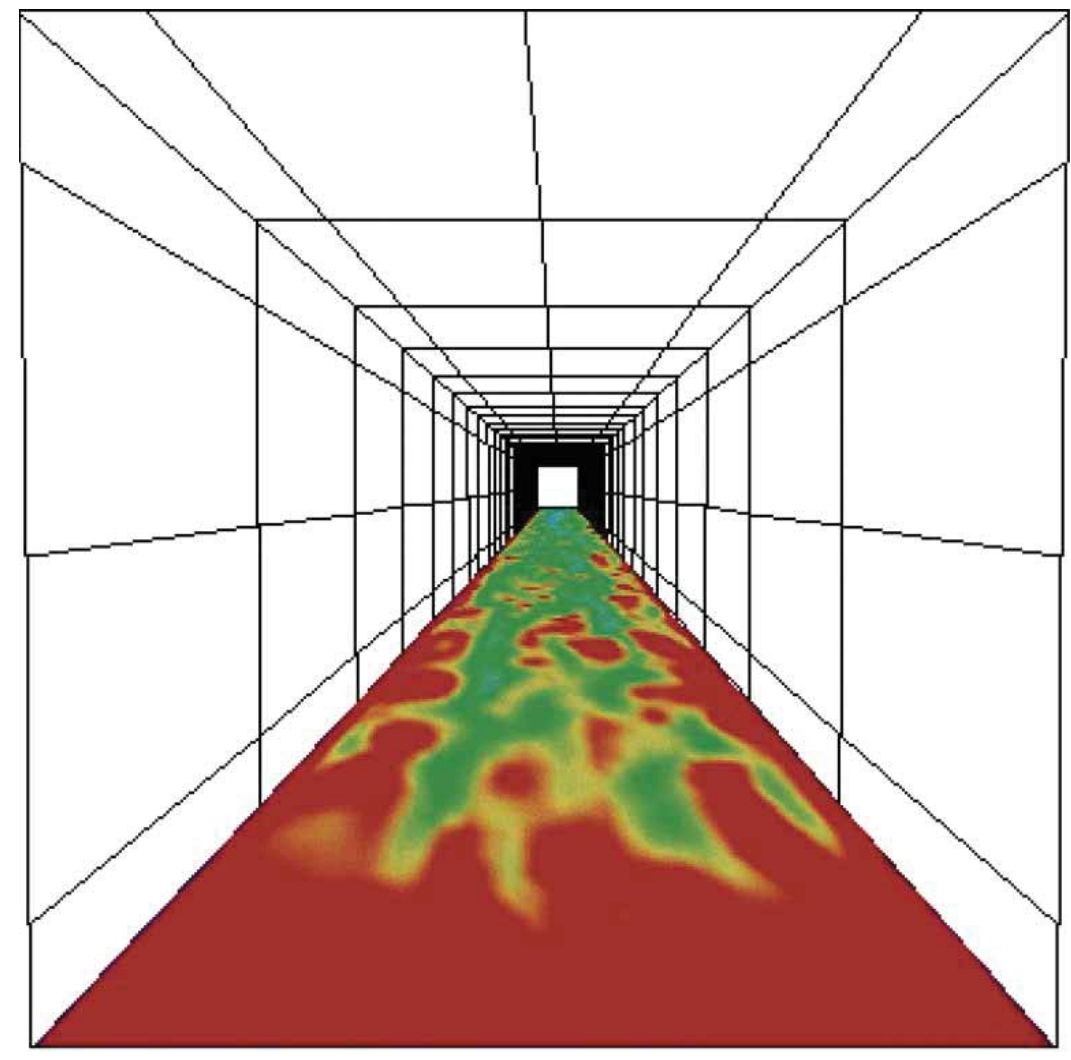

Figure 15. Heat flux at the heated wall (LES-temp). Red colours correspond to strong heat flux and blue to low heat flux. Click here for animation. 


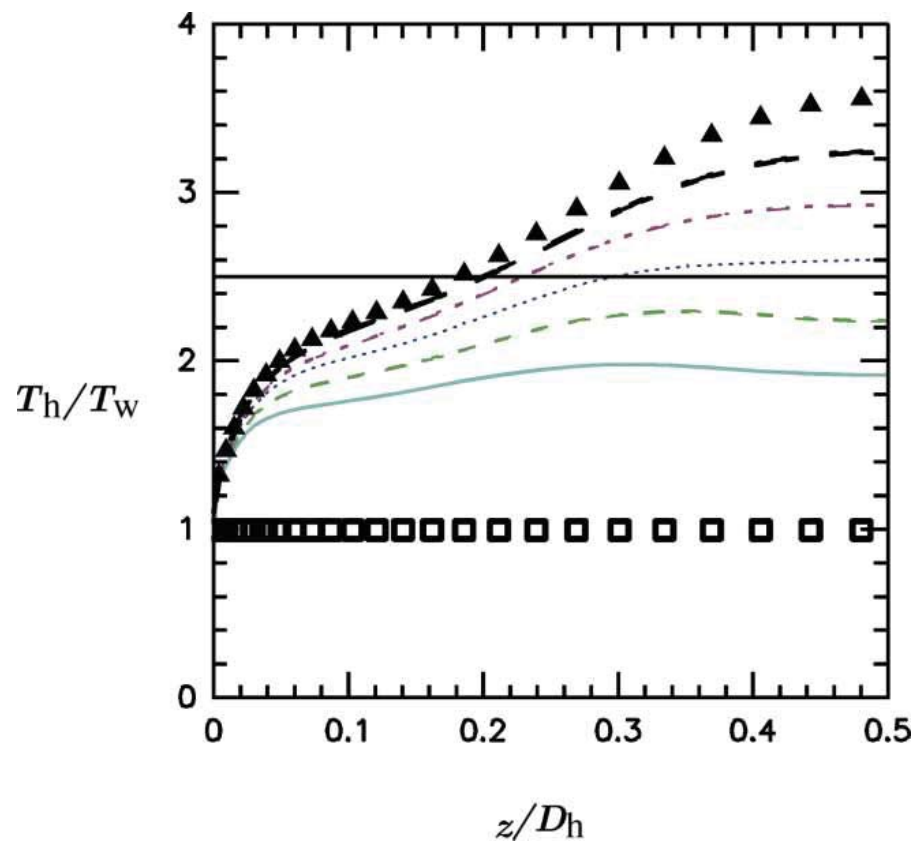

Figure 16. Profiles of $T_{\mathrm{h}} / T_{\mathrm{W}}$ at seven different $x$-planes of the LES-flux: $x / D_{\mathrm{h}}=0, \square ; x / D_{\mathrm{h}}=5,-; x / D_{\mathrm{h}}=10$, $---; x / D_{\mathrm{h}}=15, \cdots \cdots ; x / D_{\mathrm{h}}=20,---; x / D_{\mathrm{h}}=25,--; x / D_{\mathrm{h}}=30, \boldsymbol{\Delta} ;$ LES-temp, -

weak temperature gradient. Conversely, on the external borders of the secondary flow (see figure 8), cold fluid from the duct core is transported towards the heated wall yielding a large temperature gradient and therefore locally enhanced values of the heat flux. This is associated with a local inflexion point in the profiles of $\mathrm{Nu}$ located around $\mathrm{z} / \mathrm{D}_{\mathrm{h}}=0.15$.

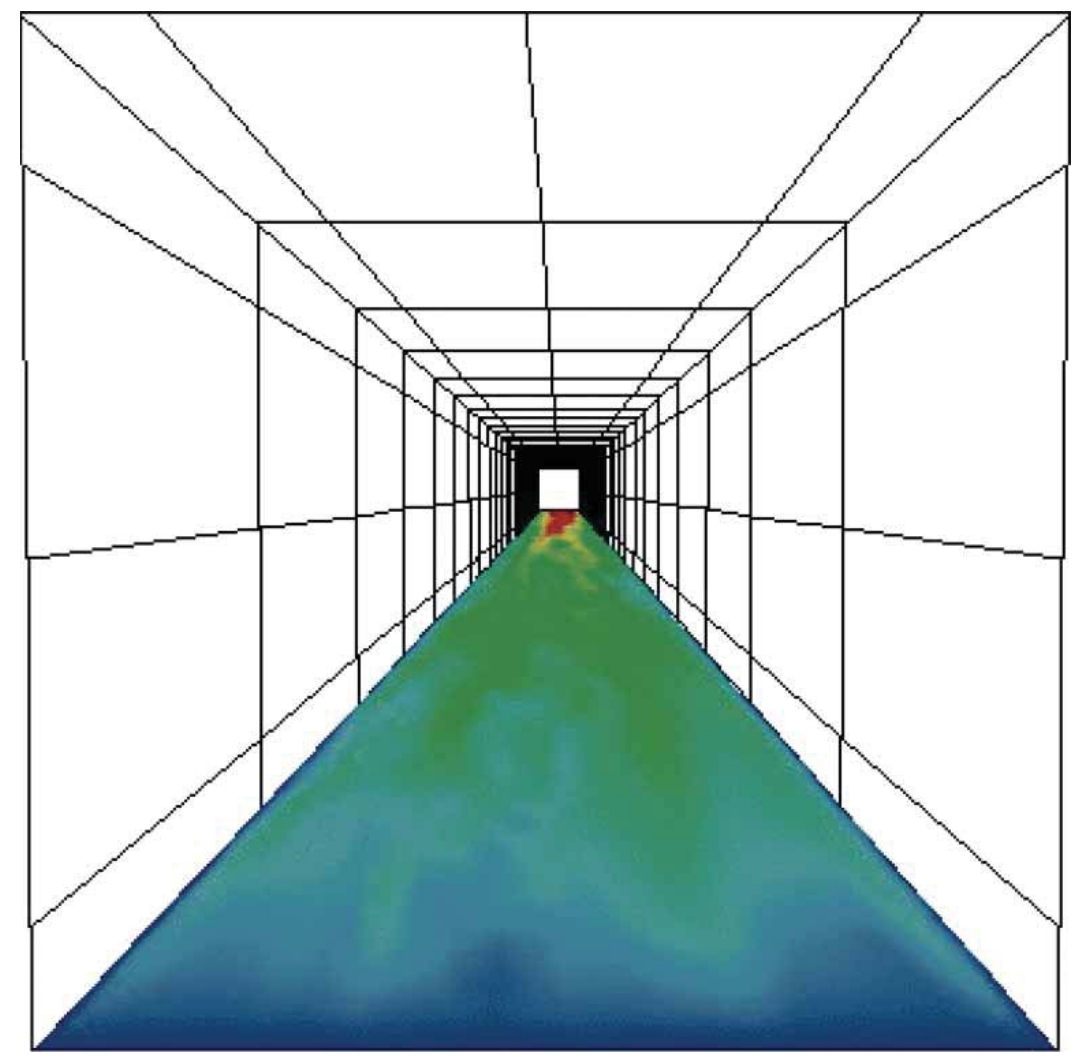

Figure 17. Instantaneous temperature at the heated wall (LES-flux). Red colours indicate maximal values and blue minimal values. Click here for animation. 
This important variation of the wall heat flux is confirmed by figure 15 which shows the instantaneous heat-flux at the heated wall. Near the duct exit, we clearly see that the low values of the wall heat flux (in blue) are concentrated near the duct middle plane when higher values are observed in the impacting regions of the secondary flow.

We now consider the LES-flux case. Figure 16 shows the variation with distance from the lateral wall of the mean temperature at the heated wall. Several downstream locations are considered here. We note the gradual increase of the temperature as we move downstream with an important growth of the thermal boundary layer in the duct middle plane. It is important to note that high values of the mean temperature are reached at $z / D_{\mathrm{h}}=0.5$ with $T_{\mathrm{h}} \approx 3.6 T_{\mathrm{w}}$ near the duct downstream end. The presence of quasi-steady secondary flows yields an important temperature variation with $z$ : similarly to the wall heat flux presented in figure 14, for large enough downstream distances the temperature profile exhibits an inflexion point near $z / D_{\mathrm{h}}=0.15$. This is associated with the impinging jets of cold fluid from the duct core situated on the external sides of the secondary vortices. Once this cold fluid has been brought near the wall, it is then transported by the secondary flow towards the duct middle plane. During this process, it is heated by the hot wall and reaches a maximum temperature in the duct middle plane before being expelled towards the duct core.

This is confirmed by figure 17 which shows the instantaneous temperature at the heated wall for the LES-flux case. Near the duct outlet, the hot regions are concentrated near the duct middle plane and very strong temperature gradients are present in the transverse direction of the heated wall.

\section{Conclusions}

Large eddy simulations have been performed to investigate the heating influence on turbulent flow within a square duct. Previous LES results [1] were obtained for a longitudinally periodic duct with a higher temperature imposed on one of the duct walls. Recent LESs performed by Hébrard et al. [2] have completed the work by Salinas-Vázquez and Métais [1] by considering the spatial development of a thermal field in a heated duct. Starting from a state where all the duct walls have the same temperature, the temperature of one of the walls is suddenly increased at a given downstream location. It has been shown that the boundary conditions proposed by Poinsot and Lele [3] based on characteristic waves and aimed at the simulation of spatially developing flows provide good results in the case of the heated duct. To simulate flows in closer correspondence with industrial situations, previous results [2] were compared with a case for which a constant heat flux was imposed on the heated wall. For these two spatially growing cases, we observed similar trends to the fully developed situation investigated in [1]. Further downstream, we noted the progressive reinforcement and growth of secondary flow near the heated wall and the formation of a unique ejection of hot fluid near the latter. Despite the relatively important length of the spatial duct (more than 30 hydraulic diameters), the thermal state is very different from the fully developed thermal state reached in the periodic case. In particular, the ejection is reduced both in size and intensity. Furthermore, we have shown that the secondary flows play a major role in the distribution of heat flux and temperature at the heated wall. Due to the presence of these secondary vortices, the thermal characteristics are found to vary significantly in the transverse direction of the heated wall. This may have very important implications in industrial heat exchangers since these strong thermal variations cause important mechanical stress within the duct wall materials. 


\section{Acknowledgement}

All the numerical computations were carried out at the Institut du Développement et des Ressources en Informatique Scientifique.

\section{References}

[1] Salinas-Vázquez, M. and Métais, O., 2002, Large eddy simulation of the turbulent flow through a heated square duct. Journal of Fluid Mechanics, 453, 201-238.

[2] Hébrard, J., Salinas-Vázquez, M. and Métais, O., 2004, Large eddy simulation of turbulent duct flow: heating and curvature effects. Journal of Heat Fluid Flow, 25, 569-580.

[3] Poinsot, T. and Lele, S., 1992, Boundary conditions for direct simulations of compressible viscous flows. Journal of Computational Physics, 101, 104-129.

[4] Grinstein, F.F., 1994, Open boundary conditions in the simulation of subsonic turbulent shear flows. Journal of Computational Physics, 115, 43-55.

[5] Ducros, F., Comte, P. and Lesieur, M., 1996, Large-eddy simulation of transision to turbulence in a boundarylayer developing spatially over a flat plate. Journal of Fluid Mechanics, 326, 1-36.

[6] White, F.M., 1991, Viscous Fluid Flow (New York: McGraw-Hill).

[7] Lesieur, M. and Métais, O., 1996, New trends in large eddy simulations of turbulence. Annual Review of Fluid Mechanics, 28 45-82.

[8] Lesieur, M. and Comte, P., 1997, Large-eddy simulations of compressible turbulent flows. AGARD-VKI course: Turbulence in Compressible Flows, AGARD report 819.

[9] Métais, O., Lesieur, M. and Comte, P., 1999, Large-eddy simulations of incompressible and compressible turbulence. In: A. Hanifi et al. (Eds), Transition, Turbulence and Combustion Modelling (Dordrecht: Kluwer).

[10] Favre, A., 1965, Equations des gaz turbulentes compressibles. ii-Méthode des vitesses macroscopiques pondérés par la mass volumique Journal de mécanique, 4, 391-421.

[11] Gottlieb, D. and Turkel, E., 1976, Dissipative two four methods for time-dependent problems. Mathématics Computation, 30, 703-723.

[12] Kennedy, C.A. and Carpenter, M.H., 1997, Comparison of several numerical methods for simulation of compressible shear layers. NASA Technical Paper, 3484.

[13] Hixon, T. and Turkel, E., 1999, Compact implicit MacCormack-type schemes with high accuracy. Journal of Computational Physics, 158, 51-70.

[14] Poinsot, T. and Veynante, D., 2001, Theoretical and Numerical Combustion (USA: Edwards).

[15] Hunt, J., Wray, A. and Moin, P., 1988, Eddies, stream, and convergence zones in turbulent flows. Center for Turbulence Research Rep CTR-S88.

[16] Dubief, Y. and Delcayre, F., 2000, On coherent-vortex identification in turbulence. Journal of Turbulence, 1, 11.

[17] Jones, O.C., 1976, An improvement in the calculation of turbulent friction in rectangular ducts. Transactions of ASME: Journal of Fluid Engineering, 98, 173-181.

[18] Gavrilakis, S., 1992, Numerical simulation of low Reynolds number turbulent flow through a straight square duct. Journal of Fluid Mechanics, 244, 101-112. 